Letter Report

\title{
Yucca Mountain Environmental Monitoring Systems Initiative Air Quality Scoping Study for Crater Flat, Nye County, Nevada
}

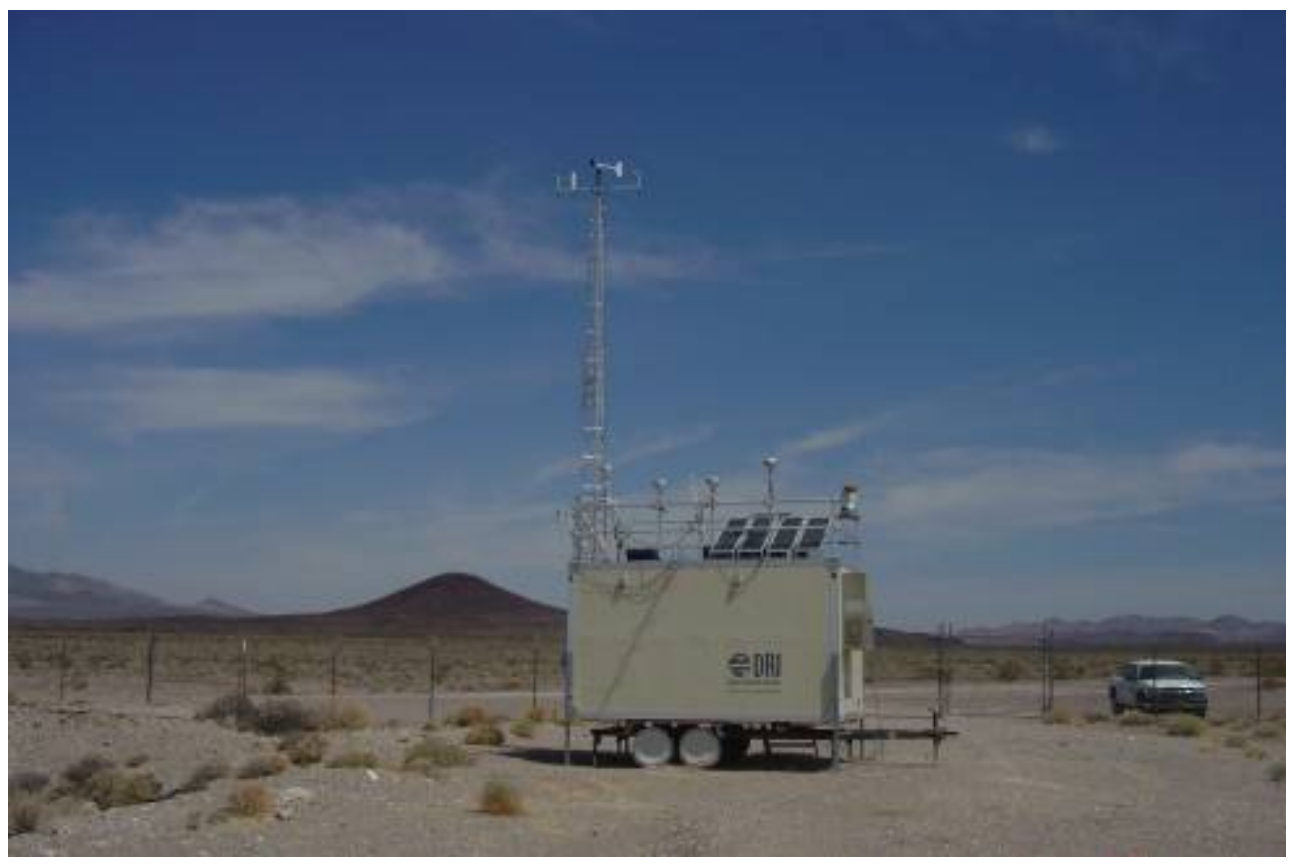

prepared by

Johann Engelbrecht, Ilias Kavouras, Dave Campbell, Scott Campbell, Steven Kohl and David Shafer

Desert Research Institute

Nevada System of Higher Education

submitted to

Nevada Site Office

National Nuclear Security Administration

U.S. Department of Energy

Las Vegas, Nevada

August 2008

The work upon which this report is based was supported by the U.S. Department of Energy under Contract \#DE-AC52-06NA26383. 
Reference herein to any specific commercial product, process, or service by trade name, trademark, manufacturer, or otherwise, does not necessarily constitute or imply its endorsement, recommendation, or favoring by the United States Government or any agency thereof or its contractors or subcontractors. The views and opinions of authors expressed herein do not necessarily state or reflect those of the United States Government of any agency thereof.

This report has been reproduced directly from the best available copy.

Available for sale to the public, if paper, from:

\author{
U.S. Department of Commerce \\ National Technical Information Service \\ 5285 Port Royal Road \\ Springfield, VA 22161 \\ Phone: 800.553.6847 \\ Fax: 703.605.6900 \\ Email: orders@ntis.gov \\ Online ordering: http://www.ntis.gov/ordering.htm
}

Available electronically at http://www.osti.gov/bridge.

Available for a processing fee to the U.S. Department of Energy and its contractors, in paper, from:

U.S. Department of Energy

Office of Scientific and Technical Information

P.O. Box 62

Oak Ridge, TN 37831-0062

Phone: 865.576.8401

Fax: 865.576.5728

Email: reports@adonis.osti.gov 


\section{CONTENTS}

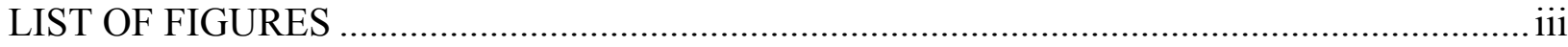

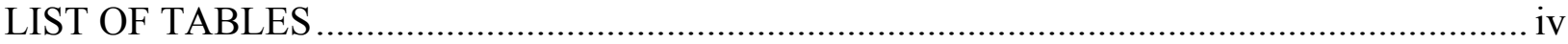

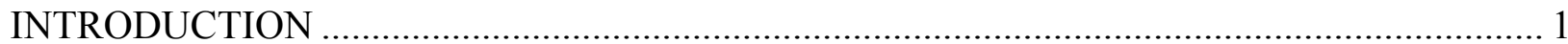

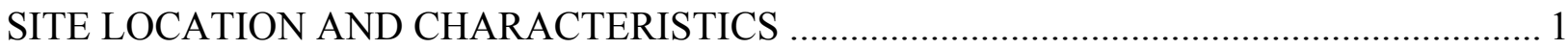

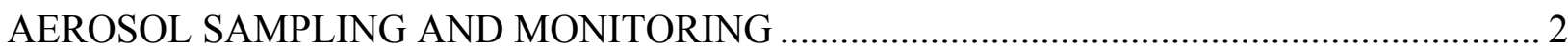

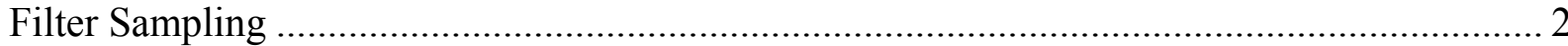

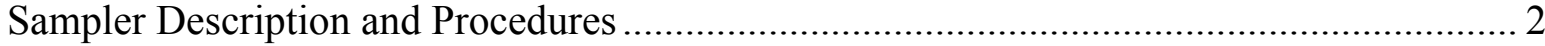

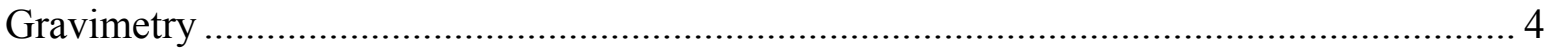

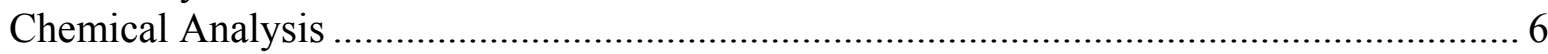

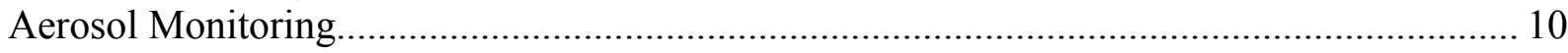

Monitor Description and Procedures ………………………....................................... 10

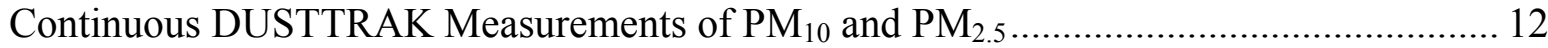

Comparison of Filter to Continuous Results...................................................................... 14

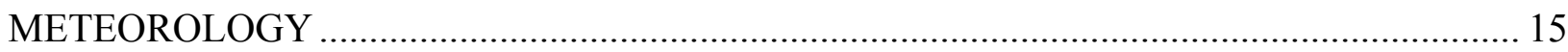

Relationships of Meteorology with Aerosol Measurements.................................................... 19

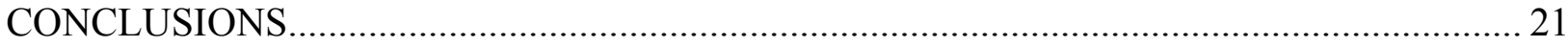

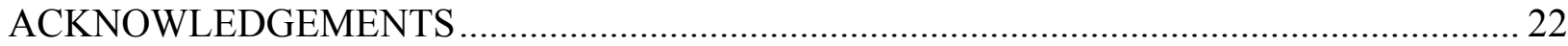

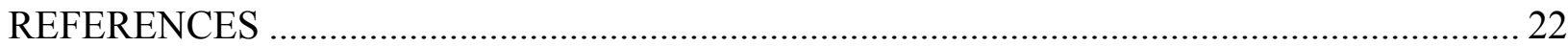

\section{LIST OF FIGURES}

1. Southern Nevada map showing the location of Site \#7 (at Crater Flat), Nevada Test Site, and Yucca Mountain.

2. Photographs of PQ100 (green/gray box in left photo), PQ200 (white box in left photo) and their sampling inlets (right photo)............................................................................. 2

3. A diagrammatic representation of the BGI $\mathrm{PM}_{2.5}$ sampler showing the $\mathrm{PM}_{10}$ size selective impactor head as the first stage followed by a $\mathrm{PM}_{2.5}$ VSCC. ............................................... 3

4. Time series of $\mathrm{PM}_{10}$ and $\mathrm{PM}_{2.5}$ mass concentrations ( \pm uncertainty) at Site \#7 (Crater

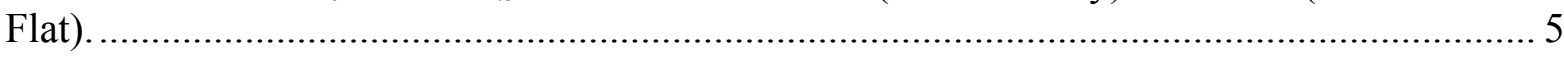

5. Relationship between mean ( \pm uncertainty) daily $\mathrm{PM}_{2.5}$ and $\mathrm{PM}_{10}$ at Crater Flat................... 6

6. Reconstructed mass for PM10 and PM2.5 based on chemical composition. ........................ 10

7. Left photograph: The front panels of $\mathrm{PM}_{10}$ (right on the left photograph) and $\mathrm{PM}_{2.5}$ (left on the left photograph) of TEOM. Right photograph: The DUSTTRAK monitors (green)

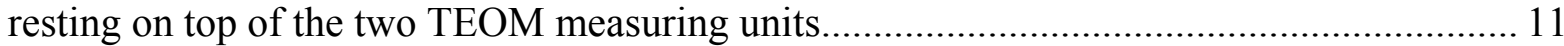

8. Schematic drawing of the sampling inlet for the DUSTTRAK (not to scale)........................ 12

9. Mean 24-h $\mathrm{PM}_{10}$ and $\mathrm{PM}_{2.5}$ mass concentrations measured by DUSTTRAK at Site \#7

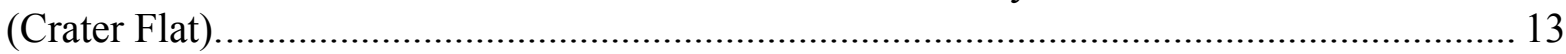

10. $\mathrm{PM}_{2.5} / \mathrm{PM}_{10}$ mass ratios at Site \#7 (Crater Flat) ................................................................ 13

11. Variation of mean $\left( \pm\right.$ st.error) $\mathrm{PM}_{10}$ and $\mathrm{PM}_{2.5}\left(\mu \mathrm{g} / \mathrm{m}^{3}\right)$ in weekdays and weekends at Site \#7 (Crater Flat) (Monday $=1$, Tuesday $=2$, Wednesday $=3$, Thursday $=4$, Friday $=5$, Saturday $=6$, Sunday $=7$ ). 
12. Relationships between $\mathrm{PM}_{10}$ concentrations $\left(\mu \mathrm{g} / \mathrm{m}^{3}\right)$ measured by DUSTTRAK, and filter-based methods.

13. Relationships between $\mathrm{PM}_{2.5}$ concentrations $\left(\mu \mathrm{g} / \mathrm{m}^{3}\right)$ measured by DUSTTRAK, and filter-based methods

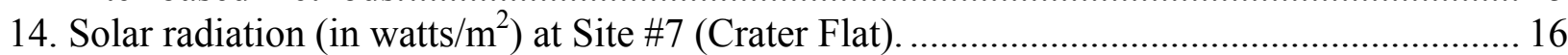

15. Temperature (in ${ }^{\circ} \mathrm{F}$ ) and relative humidity at Site \#7 (Crater Flat)................................... 16

16. Wind speed (in miles/hr) at Site \#7 (Crater Flat)......................................................... 17

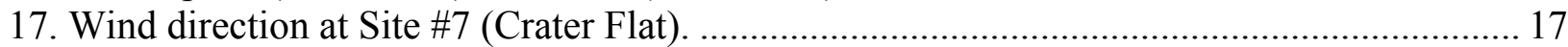

18. Wind direction and speed at Crater Flat. .................................................................. 18

19. Average wind speed for each wind direction sector................................................. 19

20. Hourly variation of $\mathrm{PM}_{10}$ and $\mathrm{PM}_{2.5}$ mass concentrations $\left(\mu \mathrm{g} / \mathrm{m}^{3}\right)$ as well as wind speed (miles/hour) at Site \#7 (Crater Flat)............................................................................. 20

21. Mean ( \pm st.error) of $\mathrm{PM}_{10}$ mass concentrations $\left(\mu \mathrm{g} / \mathrm{m}^{3}\right)$ for different wind direction sectors at Site \#7 (Crater Flat). ................................................................................... 20

22. Mean ( \pm st.error) of $\mathrm{PM}_{2.5}$ mass concentrations $\left(\mu \mathrm{g} / \mathrm{m}^{3}\right)$ for different wind direction sectors at Site \#7 (Crater Flat).

\section{LIST OF TABLES}

1. Longitude, latitude, and elevation of the mobile trailer location at Site \#7 (Crater Flat)........ 2

2. Collection days, filter numbers, mass, and uncertainties by gravimetric analysis, and associated flags of samples from Site \#7 (Crater Flat).

3. Results of the chemical analysis for selected filters from Crater Flat. Chemical components with concentrations higher than twice the uncertainty are in bold, while those with concentrations lower than twice the uncertainty are in italics. Concentrations are in $\mu \mathrm{g} / \mathrm{m}^{3}$

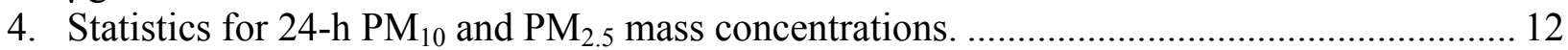

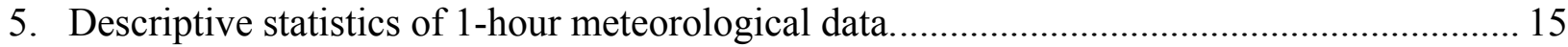

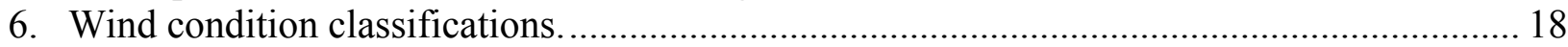




\section{INTRODUCTION}

The Desert Research Institute (DRI) is performing a scoping study as part of the U.S. Department of Energy's Yucca Mountain Environmental Monitoring Systems Initiative (EMSI). The main objective is to obtain baseline air quality information for Yucca Mountain and an area surrounding the Nevada Test Site (NTS).

Air quality and meteorological monitoring and sampling equipment housed in a mobile trailer (shelter) (cover page figure) is collecting data at eight sites outside the NTS, including Ash Meadows National Wildlife Refuge (NWR), Beatty, Sarcobatus Flats, Rachel, Caliente, Pahranagat NWR, Crater Flat, and Tonopah Airport, and at four sites on the NTS (Engelbrecht et al., 2007a-d). The trailer is stationed at any one site for approximately eight weeks at a time.

This letter report provides a summary of air quality and meteorological data, on completion of the site's sampling program.

\section{SITE LOCATION AND CHARACTERISTICS}

Crater Flat is adjacent to the proposed Yucca Mountain nuclear repository, just outside of the NTS boundaries. The existing plans for the facility indicate that a railroad and heavy-haul roads, to transfer nuclear waste to the repository, will pass through Crater Flat. The sampling site is located 15 miles southeast of the town of Beatty, and about 6 miles east of U.S. Route 95. It is about 90 miles northwest of Las Vegas (Figure 1).

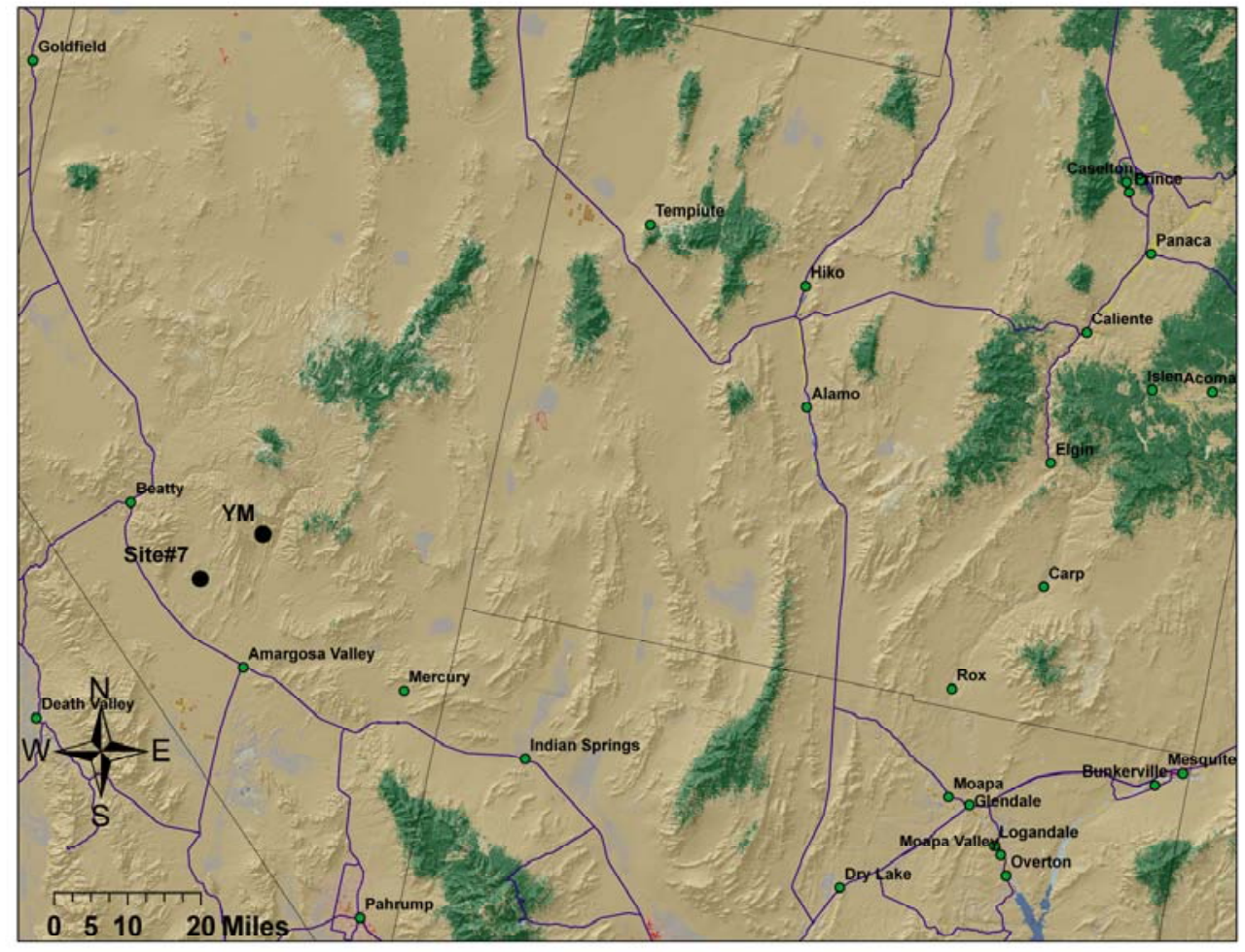

Figure 1. Southern Nevada map showing the location of Site \#7 (at Crater Flat), Nevada Test Site, and Yucca Mountain. The map background is land use and land cover from the 2001 National Land Cover Database. 
The mobile trailer was located inside a fenced area at well VH-1, on the edge of the Yucca Mountain Repository Facility Program area. Monitoring of $\mathrm{PM}_{10}, \mathrm{PM}_{2.5}$, and meteorological conditions was carried out from May 25, 2007, to August 29, 2007. Because of the remote location of the site, instrumentation that required 120-volt AC power (TEOMs) did not run. The portable DUSTTRAK, meteorological equipment, and integrated PM samplers were run from 12-volt batteries, charged by solar panels.

Table 1. Longitude, latitude, and elevation of the mobile trailer location at Site \#7 (Crater Flat).

\begin{tabular}{cc}
\hline Site & Crater Flat \\
\hline Latitude & $36^{\circ} 47^{\prime} 31.81^{\prime \prime}$ \\
Longitude & $116^{\circ} 33^{\prime} 9.75^{\prime \prime}$ \\
\hline
\end{tabular}

\section{AEROSOL SAMPLING AND MONITORING}

\section{Filter Sampling}

\section{Sampler Description and Procedures}

BGI, Inc., PQ100 and PQ200 Ambient PM 2.5 Federal Reference Method (FRM) samplers were used to collect 24-h integrated $\mathrm{PM}_{10}$ and $\mathrm{PM}_{2.5}$ samples. Figure 2 shows the PQ100 and PQ200 in the mobile trailer (left) and the $\mathrm{PM}_{10}$ sampling inlets on the top of the trailer (right). Both the PQ100 (Designation No. RFPS-1298-124) and PQ200 (Designation No. RFPS-0498-116) samplers are designed to meet the criteria for collecting 24-h samples of ambient aerosol according to the U.S. National Ambient Air Quality Standards (NAAQS).

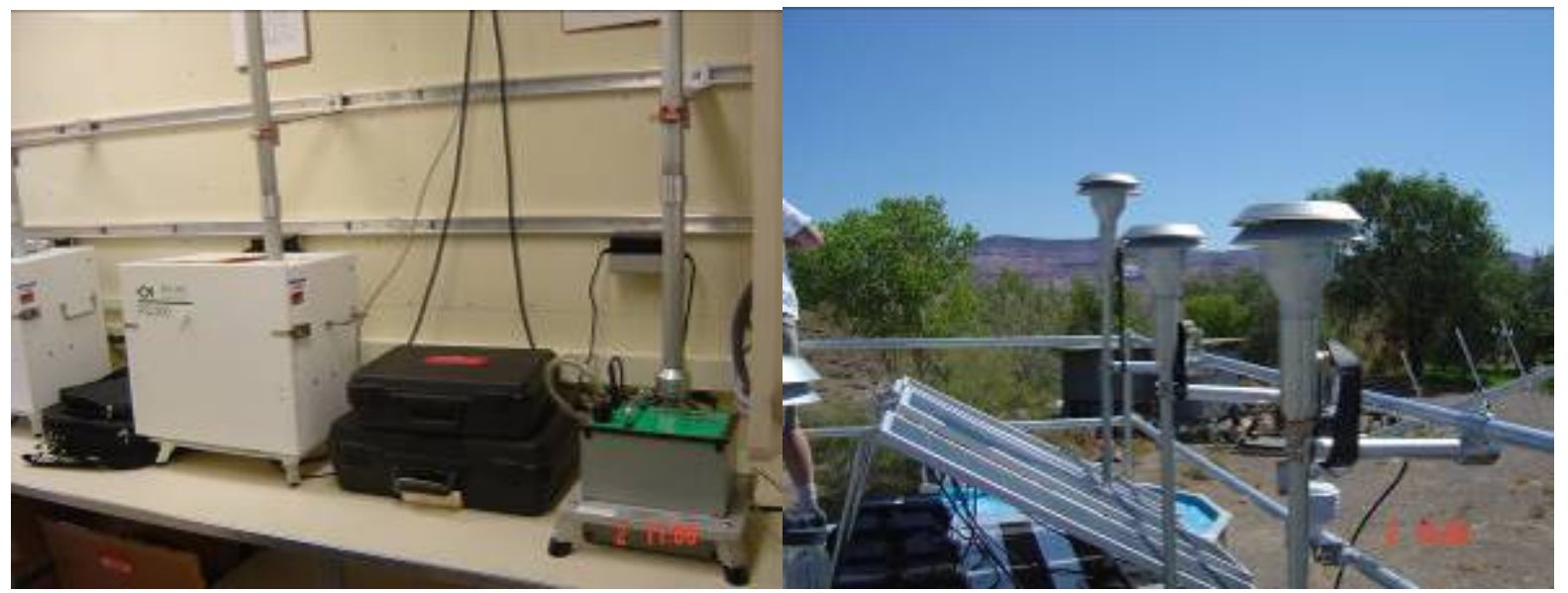

Figure 2. Photographs of PQ100 (green/gray box in left photo), PQ200 (white box in left photo) and their sampling inlets (right photo).

Figure 3 shows a schematic drawing of the samplers. Particles with aerodynamic diameter larger than $10 \mu \mathrm{m}$ are removed by impaction by the size selective inlet, while the smaller particles remain airborne. The $\mathrm{PM}_{10}$ fraction is collected by a filter located downstream of the size selective inlet. For the collection of $\mathrm{PM}_{2.5}$, particles in the range between 2.5 and $10 \mu \mathrm{m}$ were removed by the Very Sharp Cut Cyclone (VSCC) (U.S. 
Environmental Protection Agency [EPA] Equivalent Designation No. EQPM-0202-142), then collected on a filter.

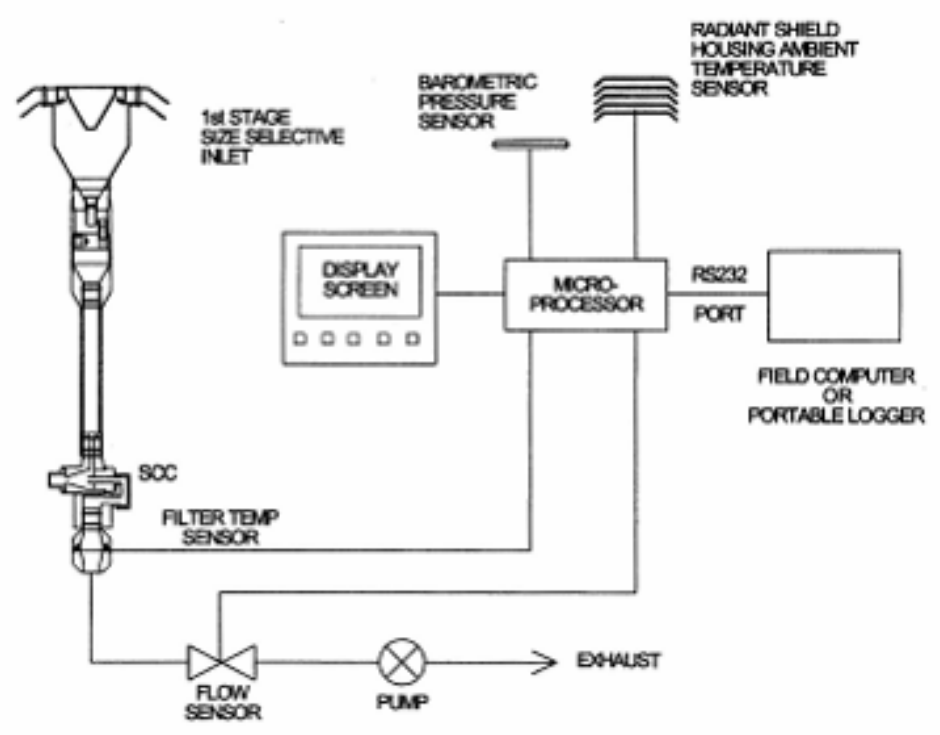

Figure 3. A diagrammatic representation of the $\mathrm{BGI} \mathrm{PM}_{2.5}$ sampler showing the $\mathrm{PM}_{10}$ size selective impactor head as the first stage followed by a $\mathrm{PM}_{2.5}$ VSCC. This configuration can be readily modified to a $\mathrm{PM}_{10}$ sampler by removal of the VSCC.

For both PQ100 and PQ200, samples were collected at a volumetric flow rate of 16.67 liters $/ \mathrm{min}$. The flow rate is controlled to \pm 2 percent precision with a mass flow controller. The actual ambient temperature and barometric pressure, filter temperature and pressure, and anomalies (if any) were recorded by a microprocessor. The sampler was equipped to operate from an internal 12-volt DC battery. The battery was normally recharged from 120-volt AC. Alternatively, a 32-watt solar panel with an additional external ballast battery was installed to provide power for periods without electricity. Two sets of PQ100 and PQ200 samplers were installed in the mobile trailer. $\mathrm{PM}_{10}$ and $\mathrm{PM}_{2.5}$ samples were collected on filters in numbered cassettes, labeled TT (for PM 10 Teflon), FT (for $\mathrm{PM}_{2.5}$ Teflon), TQ (for PM $\mathrm{M}_{10}$ Quartz), and FQ (for $\mathrm{PM}_{2.5}$ Quartz). Each filter cassette was loaded with a preweighed 46.2-mm-diameter PTFE (Teflon) membrane filter (Whatman \# 7592-004) or 47mm quartz fiber (Pallflex \#2500QAT-UP) filter. The Teflon membrane collected particles for gravimetric analysis, light absorption by densitometry, and elements by X-ray fluorescence spectrometry. Quartz fiber filters were used for measurement of water-soluble ions by atomic absorption spectrometry, ion chromatography, and automated colorimetry, and also for measurement of carbon species by thermal optical reflectance.

Operation, calibration, and maintenance of PQ100 and PQ200 particulate samplers are described in standard operating procedure DRI SOP \# 1-211.2 "BGI PQ100 PM10 and PQ200 PM2.5 REFERENCE SAMPLERS FOR THE YUCCA MOUNTAIN AIR QUALITY PROGRAM." Flow calibration and leak tests (only for PQ200) were performed on the day of installation (May 25, 2007). The leak check was performed according to the manufacturer's operational instruction manual only for PQ200; no procedure exists from the manufacture for the PQ100. The flow rates were set according to a BGI Tri-Cal NIST 
traceable standard. The sampler was then placed in "calibration" or "run" mode and a onepoint calibration verification or one-point flow-rate verification performed. Aerosol samples were collected on a 1-in-6-day schedule. Audits of the flow and leak tests were done onsite at the beginning and end of the monitoring campaign. Teflon and quartz filters were prepared and assembled in their filter holders by the Desert Research Institute's (DRI) Environmental Analysis Facility (EAF) in Reno and shipped to DRI's facilities in Las Vegas. The filters were kept at $-4^{\circ} \mathrm{C}$ and transported to the field in a cryo-cooler. Exposed filters were also stored at $-4^{\circ} \mathrm{C}$ in Las Vegas. Upon completion of the monitoring period at the site, all filters were shipped to the EAF in Reno.

\section{Gravimetry}

Table 2 shows mass concentrations (and uncertainty) of filters collected at Crater Flat. $\mathrm{PM}_{10}$ mass concentrations varied from $5.24 \mu \mathrm{g} / \mathrm{m}^{3}$ to $27.62 \mu \mathrm{g} / \mathrm{m}^{3}$, while $\mathrm{PM}_{2.5}$ mass concentrations ranged from $2.99 \mu \mathrm{g} / \mathrm{m}^{3}$ to $14.31 \mu \mathrm{g} / \mathrm{m}^{3}$. Similar temporal trends were observed for both $\mathrm{PM}_{10}$ and $\mathrm{PM}_{2.5}$. In all cases, 24-h $\mathrm{PM}_{10}$ and $\mathrm{PM}_{2.5}$ levels were significantly lower than the NAAQS as recently revised by EPA $\left(24-\mathrm{h}\right.$ PM $_{10}: 150 \mu \mathrm{g} / \mathrm{m}^{3}$, 24-h $\mathrm{PM}_{2.5}: 35 \mu \mathrm{g} / \mathrm{m}^{3}$; Annual $\mathrm{PM}_{2.5}: 15 \mu \mathrm{g} / \mathrm{m}^{3}$ ) (Figure 4). On average, fine particulates $\left(\mathrm{PM}_{2.5}\right)$ accounted for approximately half of $\mathrm{PM}_{10}\left(\mathrm{PM}_{2.5} / \mathrm{PM}_{10}\right.$ ratio of 0.49$)$ (Figure 5). This value indicated the significant contribution of coarse particles probably due to the emissions from nearby unpaved roads that connect several mine operations with U.S. Route 95. The possible contributions of smoke from a human-ignited fire (Zaca) in Santa Barbara, California, may have contributed to high $\mathrm{PM}_{10}$ and $\mathrm{PM}_{2.5}$ levels. The fire ignited on July 4 , 2008, and lasted more than two months.

Table 2. Collection days, filter numbers, mass, and uncertainties by gravimetric analysis, and associated flags of samples from Site \#7 (Crater Flat).

\begin{tabular}{|c|c|c|c|c|c|}
\hline Date & No & Type & $\begin{array}{l}\text { Mass } \\
\left(\mu \mathrm{g} / \mathrm{m}^{3}\right) \\
\end{array}$ & $\begin{array}{l}\text { Uncertainty } \\
\left(\mu \mathrm{g} / \mathrm{m}^{3}\right)\end{array}$ & Flags \\
\hline \multirow[t]{2}{*}{$5 / 30 / 2007$} & \multirow[t]{2}{*}{085} & $\mathrm{PM}_{10}$ & -99.0000 & -99.0000 & $\begin{array}{l}\text { V: invalid (void) analysis result; } \\
\text { T: sampling time error }\end{array}$ \\
\hline & & $\mathrm{PM}_{2.5}$ & 6.2422 & 0.4456 & \\
\hline \multirow{2}{*}{$6 / 05 / 2007$} & \multirow{2}{*}{087} & $\mathrm{PM}_{10}$ & 27.6206 & 0.6986 & \\
\hline & & $\mathrm{PM}_{2.5}$ & 14.3154 & 0.5148 & \\
\hline \multirow{2}{*}{$6 / 11 / 2007$} & \multirow{2}{*}{088} & $\mathrm{PM}_{10}$ & 15.3910 & 0.5269 & $\mathrm{~N}$ : foreign substance on sample \\
\hline & & $\mathrm{PM}_{2.5}$ & 9.0266 & 0.4641 & \\
\hline \multirow{2}{*}{$6 / 17 / 2007$} & & $\mathrm{PM}_{10}$ & 13.1448 & 0.5020 & \\
\hline & & $\mathrm{PM}_{2.5}$ & 5.5347 & 0.4419 & \\
\hline \multirow{2}{*}{$6 / 23 / 2007$} & & $\mathrm{PM}_{10}$ & -99.0000 & -99.0000 & V: invalid (void) analysis result \\
\hline & & $\mathrm{PM}_{2.5}$ & -99.0000 & -99.0000 & $\mathrm{~V}$ : invalid (void) analysis result \\
\hline \multirow{2}{*}{$6 / 29 / 2007$} & & $\mathrm{PM}_{10}$ & 11.6889 & 0.4873 & \\
\hline & & $\mathrm{PM}_{2.5}$ & 5.9068 & 0.4436 & \\
\hline \multirow{2}{*}{$7 / 05 / 2007$} & & $\mathrm{PM}_{10}$ & 8.4443 & 0.4598 & \\
\hline & & $\mathrm{PM}_{2.5}$ & 4.0349 & 0.4352 & \\
\hline \multirow{2}{*}{$7 / 11 / 2007$} & & $\mathrm{PM}_{10}$ & 18.7188 & 0.5683 & $\mathrm{~N}$ : foreign substance on sample \\
\hline & & $\mathrm{PM}_{2.5}$ & 8.2779 & 0.4585 & \\
\hline \multirow{2}{*}{$7 / 17 / 2007$} & & $\mathrm{PM}_{10}$ & 12.7288 & 0.4977 & $\mathrm{~N}$ : foreign substance on sample \\
\hline & & $\mathrm{PM}_{2.5}$ & 7.0715 & 0.4504 & \\
\hline
\end{tabular}


Table 3. Collection days, filter numbers, mass, and uncertainties by gravimetric analysis, and associated flags of samples from Site \#7 (Crater Flat) (continued).

\begin{tabular}{ccccc}
\hline Date & No & Type & $\begin{array}{c}\text { Mass } \\
\left(\mu \mathrm{g} / \mathrm{m}^{3}\right)\end{array}$ & $\begin{array}{c}\text { Uncertainty } \\
\left(\mu \mathrm{g} / \mathrm{m}^{3}\right)\end{array}$ \\
\hline $7 / 23 / 2007$ & $\mathrm{PM}_{10}$ & 23.5827 & 0.6367 & N: foreign substance on sample \\
& $\mathrm{PM}_{2.5}$ & 8.2363 & 0.4582 & \\
$7 / 29 / 2007$ & $\mathrm{PM}_{10}$ & 14.3095 & 0.5145 & \\
& $\mathrm{PM}_{2.5}$ & 7.9867 & 0.4565 & \\
$8 / 04 / 2007$ & $\mathrm{PM}_{10}$ & 21.7554 & 0.6101 & N: foreign substance o n sample \\
& $\mathrm{PM}_{2.5}$ & 11.6473 & 0.4869 & \\
$8 / 10 / 2007$ & $\mathrm{PM}_{10}$ & 13.1448 & 0.5020 & \\
& $\mathrm{PM}_{2.5}$ & 6.0732 & 0.4445 & \\
$8 / 16 / 2007$ & $\mathrm{PM}_{10}$ & 13.0616 & 0.5011 & \\
& $\mathrm{PM}_{2.5}$ & 9.9834 & 0.4719 & \\
$8 / 22 / 2007$ & $\mathrm{PM}_{10}$ & 19.7171 & 0.5817 & \\
& $\mathrm{PM}_{2.5}$ & 8.1981 & 0.4581 & \\
& $\mathrm{PM}_{10}$ & 5.2413 & 0.4403 & \\
\hline
\end{tabular}

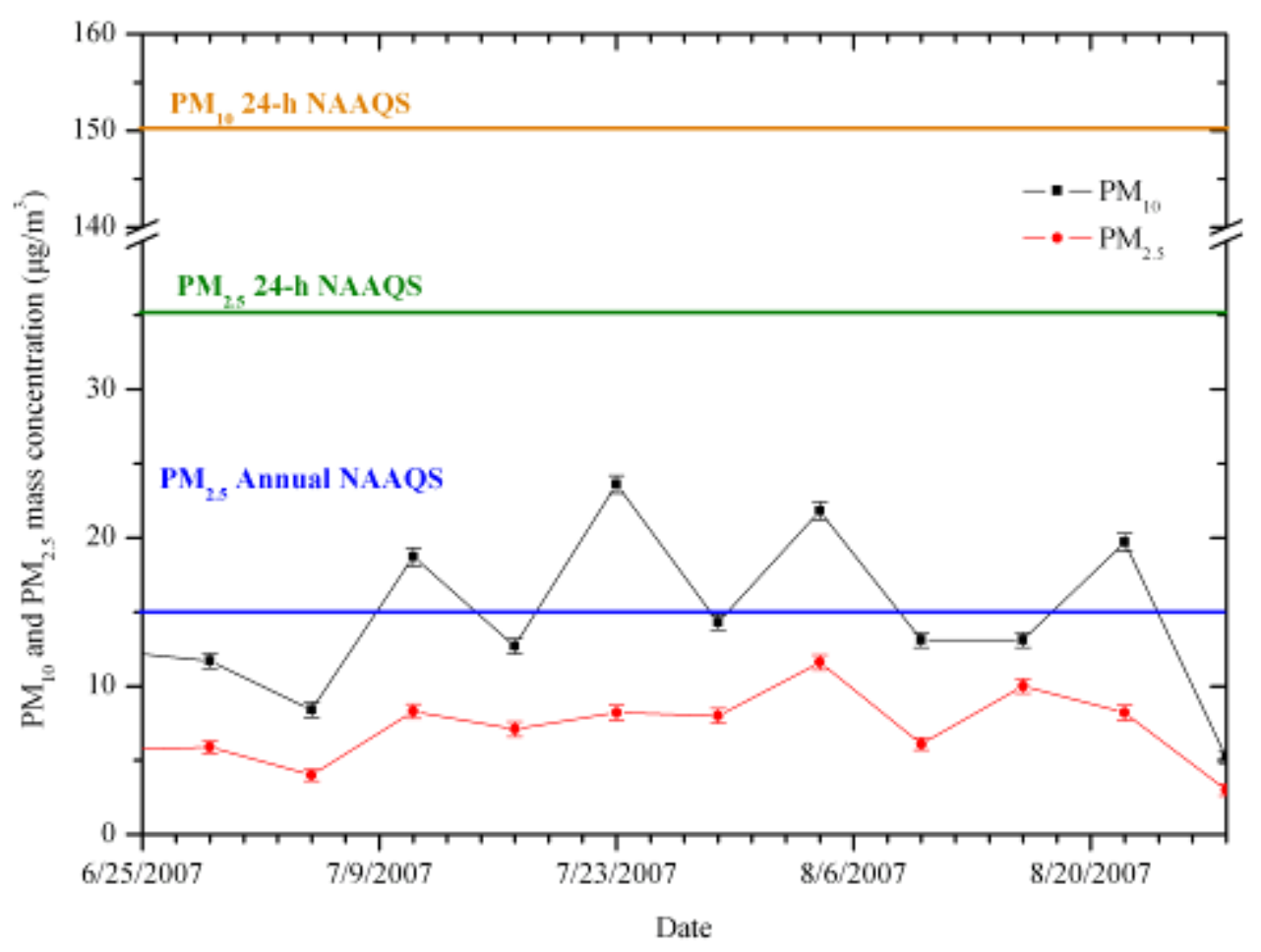

Figure 4. Time series of $\mathrm{PM}_{10}$ and $\mathrm{PM}_{2.5}$ mass concentrations ( \pm uncertainty) at Site \#7 (Crater Flat). 


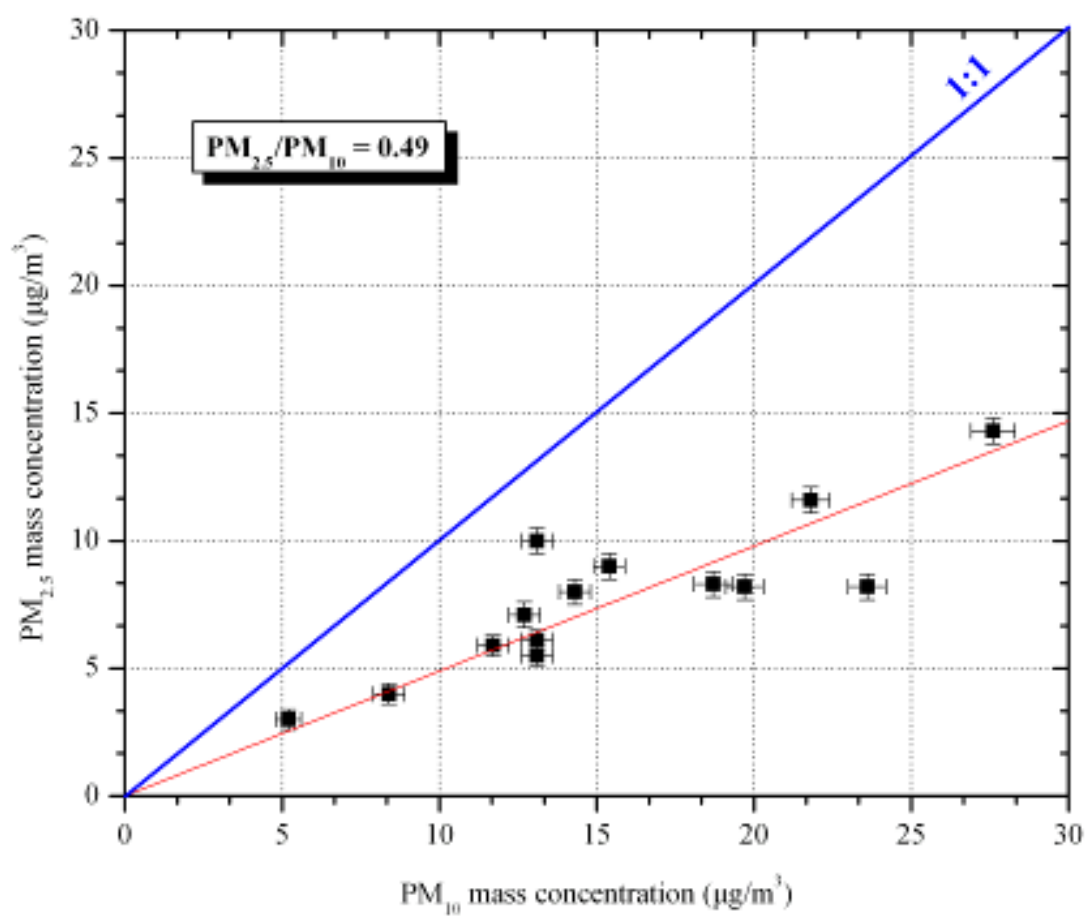

Figure 5. Relationship between mean ( \pm uncertainty) daily $\mathrm{PM}_{2.5}$ and $\mathrm{PM}_{10}$ at Crater Flat.

\section{Chemical Analysis}

Table 3 shows the chemical content of $\mathrm{PM}_{10}$ and $\mathrm{PM}_{2.5}$ samples collected on June 5, 2007 and July 29, 2007. Chemical analysis included elements (from sodium to uranium) with X-ray fluorescence spectrometry (XRF), major anions (sulfate, nitrate, and chloride) by ion chromatography (IC), major cations (sodium, potassium) by atomic absorption (AA), particulate ammonium by automated colorimetry $(\mathrm{AC})$, and elemental and organic carbon by thermal optical reflectance (TOR).

Table 4. Results of the chemical analysis for selected filters from Crater Flat. Chemical components with concentrations higher than twice the uncertainty are in bold, while those with concentrations lower than twice the uncertainty are in italics. Concentrations are in $\mu \mathrm{g} / \mathrm{m}^{3}$.

\begin{tabular}{lrrrrrrrr}
\hline DATE & \multicolumn{4}{c}{$6 / 05 / 2007$} \\
\multicolumn{2}{c}{$\mathrm{PM}_{10}$} & \multicolumn{2}{c}{$\mathrm{PM}_{2.5}$} & \multicolumn{3}{c}{$\mathrm{PM}_{10}$} & \multicolumn{2}{c}{$\mathrm{PM}_{2.5}$} \\
& Conc. & Uncer. & Conc. & Uncer. & Conc. & Uncer. & Conc. & Uncer. \\
\hline Mass & $\mathbf{2 7 . 6 2 0 6}$ & $\mathbf{0 . 6 9 8 6}$ & $\mathbf{1 4 . 3 1 5 4}$ & $\mathbf{0 . 5 1 4 8}$ & $\mathbf{1 4 . 3 0 9 5}$ & $\mathbf{0 . 5 1 9 5}$ & $\mathbf{7 . 9 8 6 7}$ & $\mathbf{0 . 4 5 6 5}$ \\
\hline Chloride, $\mathrm{Cl}^{-}$ & $\mathbf{0 . 0 9 8 6}$ & $\mathbf{0 . 0 3 0 6}$ & 0.028 & 0.0295 & 0.0504 & 0.0297 & 0 & 0.0294 \\
Nitrate, $\mathrm{NO}_{3}{ }^{-}$ & $\mathbf{0 . 7 8 9 8}$ & $\mathbf{0 . 0 3 8 9}$ & $\mathbf{0 . 1 8 1 7}$ & $\mathbf{0 . 0 3 0 1}$ & $\mathbf{0 . 8 0 0 3}$ & $\mathbf{0 . 0 3 9 1}$ & $\mathbf{0 . 2 5 6}$ & $\mathbf{0 . 0 3 0 7}$ \\
Sulfate, $\mathrm{SO}_{4}{ }^{2-}$ & $\mathbf{1 . 7 2 1 8}$ & $\mathbf{0 . 0 4 7 6}$ & $\mathbf{1 . 1 7 7 2}$ & $\mathbf{0 . 0 3 9}$ & $\mathbf{1 . 5 7 1 8}$ & $\mathbf{0 . 0 4 5 1}$ & $\mathbf{1 . 3 2 9 1}$ & $\mathbf{0 . 0 4 1 3}$ \\
Ammonium, $\mathrm{NH}_{4}{ }^{+}$ & $\mathbf{0 . 4 9 9 4}$ & $\mathbf{0 . 0 3 4 5}$ & $\mathbf{0 . 4 2 6 9}$ & $\mathbf{0 . 0 3 3 3}$ & $\mathbf{0 . 5 2 5 2}$ & $\mathbf{0 . 0 3 5}$ & $\mathbf{0 . 4 5 6 7}$ & $\mathbf{0 . 0 3 3 8}$ \\
Sodium, $\mathrm{Na}^{+}$ & $\mathbf{0 . 2 6 6 1}$ & $\mathbf{0 . 0 0 8 6}$ & $\mathbf{0 . 0 5}$ & $\mathbf{0 . 0 0 5 7}$ & $\mathbf{0 . 2 7 1 8}$ & $\mathbf{0 . 0 0 8 7}$ & $\mathbf{0 . 1 6 5 7}$ & $\mathbf{0 . 0 0 6 9}$ \\
Magnesium, $\mathrm{Mg}^{2+}$ & $\mathbf{0 . 1 2}$ & $\mathbf{0 . 0 0 3 4}$ & $\mathbf{0 . 0 3 1 9}$ & $\mathbf{0 . 0 0 1 5}$ & $\mathbf{0 . 0 5 6 2}$ & $\mathbf{0 . 0 0 1 9}$ & $\mathbf{0 . 0 2 8 1}$ & $\mathbf{0 . 0 0 1 4}$ \\
Potassium, $\mathrm{K}^{+}$ & $\mathbf{0 . 2 9 9 7}$ & $\mathbf{0 . 0 0 8}$ & $\mathbf{0 . 0 7 2 7}$ & $\mathbf{0 . 0 0 3 5}$ & $\mathbf{0 . 0 9 7 2}$ & $\mathbf{0 . 0 0 3 8}$ & $\mathbf{0 . 0 6 3 7}$ & $\mathbf{0 . 0 0 3 3}$ \\
Calcium, $\mathrm{Ca}_{2+}$ & $\mathbf{3 . 0 3 4 8}$ & $\mathbf{0 . 0 7 5 4}$ & $\mathbf{0 . 4 1}$ & $\mathbf{0 . 0 1 8 3}$ & $\mathbf{0 . 4 0 5 6}$ & $\mathbf{0 . 0 1 8 2}$ & $\mathbf{0 . 1 4 2 5}$ & $\mathbf{0 . 0 1 5 7}$ \\
OC1 & $\mathbf{0 . 2 8 9 7}$ & $\mathbf{0 . 1 1 4 2}$ & $\mathbf{0 . 5 6 7 8}$ & $\mathbf{0 . 2 2 1 1}$ & $\mathbf{0 . 4 6 7 8}$ & $\mathbf{0 . 1 8 2 6}$ & $\mathbf{1 . 0 1 7 5}$ & $\mathbf{0 . 3 9 5 1}$ \\
\hline
\end{tabular}


Table 5. Results of the chemical analysis for selected filters from Crater Flat. Chemical components with concentrations higher than twice the uncertainty are in bold, while those with concentrations lower than twice the uncertainty are in italics. Concentrations are in $\mu \mathrm{g} / \mathrm{m}^{3}$ (continued).

\begin{tabular}{|c|c|c|c|c|c|c|c|c|}
\hline \multirow{3}{*}{$\begin{array}{l}\text { DATE } \\
\text { SIZE }\end{array}$} & \multicolumn{4}{|c|}{$6 / 05 / 2007$} & \multicolumn{4}{|c|}{$7 / 29 / 2007$} \\
\hline & \multicolumn{2}{|c|}{$\mathrm{PM}_{10}$} & \multicolumn{2}{|c|}{$\mathrm{PM}_{2.5}$} & \multicolumn{2}{|c|}{$\mathrm{PM}_{10}$} & \multicolumn{2}{|c|}{$\mathrm{PM}_{2.5}$} \\
\hline & Conc. & Uncer. & Conc. & Uncer. & Conc. & Uncer. & Conc. & Uncer. \\
\hline OC2 & 0.4734 & 0.1263 & 0.4045 & 0.1126 & 0.9596 & 0.2273 & 1.0903 & 0.255 \\
\hline OC3 & 1.2018 & 0.2343 & 0.5545 & 0.1764 & 1.059 & 0.2203 & 0.7917 & 0.1958 \\
\hline OC4 & 0.9126 & 0.1081 & 0.5315 & 0.0765 & 0.5889 & 0.0809 & 0.3922 & 0.0667 \\
\hline Pyrolyzed OC-TT & 1.5083 & 0.5148 & 1.1956 & 0.4087 & 0.4746 & 0.1658 & 0.2594 & 0.0958 \\
\hline Pyrolyzed OC-Op & 1.531 & 0.5445 & 1.1887 & 0.4234 & 0.3906 & 0.1435 & 0.1357 & 0.0607 \\
\hline Total OC & 4.4084 & 0.4516 & 3.247 & 0.366 & 3.4659 & 0.3816 & 3.4274 & 0.379 \\
\hline EC1 & 1.326 & 0.3031 & 1.0749 & 0.2463 & 0.5904 & 0.1372 & 0.348 & 0.0839 \\
\hline EC2 & 0.205 & 0.0801 & 0.1139 & 0.053 & 0.096 & 0.0484 & 0.0461 & 0.0383 \\
\hline EC3 & 0.0179 & 0.0115 & 0.0069 & 0.0115 & $\mathbf{0}$ & 0.0115 & $\mathbf{0}$ & 0.0115 \\
\hline Total EC & 0.0179 & 0.044 & 0.0069 & 0.0439 & 0.2957 & 0.0717 & 0.2583 & 0.0662 \\
\hline Total Carbon & 5.3485 & 0.5456 & 3.3492 & 0.3918 & 3.7851 & 0.424 & 3.6857 & 0.4166 \\
\hline $\begin{array}{l}\text { Carbonate Carbon } \\
\left(\mathrm{CO}_{3}{ }^{2-}\right)\end{array}$ & 0.9221 & 0.3424 & 0.0953 & 0.2166 & 0.0235 & 0.2149 & 0 & 0.2149 \\
\hline Sodium, $\mathrm{Na}$ & 0.2085 & 0.0835 & 0.1068 & 0.0819 & 0.1549 & 0.0827 & 0.0631 & 0.0813 \\
\hline Magnesium, Mg & 0.3547 & 0.0453 & 0.2196 & 0.0443 & 0.1242 & 0.0436 & 0.0436 & 0.0432 \\
\hline Aluminum, & 1.1168 & 0.0257 & 0.6696 & 0.0168 & 0.4276 & 0.0124 & 0.2063 & 0.009 \\
\hline Silicon, $\mathrm{Si}$ & 2.9874 & 0.0646 & 1.8426 & 0.0408 & 1.0569 & 0.0248 & 0.496 & 0.0141 \\
\hline Phosphorous, P & 0.0131 & 0.003 & 0.0108 & 0.003 & 0.0231 & 0.003 & 0.0177 & 0.003 \\
\hline Sulfur, S & 0.3513 & 0.0147 & 0.3873 & 0.0152 & 0.4995 & 0.0167 & 0.4579 & 0.0161 \\
\hline Chlorine, & 0.0158 & 0.0016 & 0.0088 & 0.0016 & 0.0083 & 0.0016 & 0.0044 & 0.0016 \\
\hline Potassium & 0.585 & 0.012 & 0.3554 & 0.00 & 0.2185 & 0.0047 & 0.0972 & 0.0025 \\
\hline Calcil & 1.0629 & 0.0215 & 0.5308 & 0.0109 & 0.4111 & 0.0086 & 0.1653 & 0.0039 \\
\hline Scandium, Sc & 0 & 0.0058 & 0 & 0.0058 & 0 & 0.0058 & 0 & 0.0058 \\
\hline Titanium, Ti & 0.0745 & 0.0019 & 0.0463 & 0.0015 & 0.04 & 0.0014 & 0.0165 & 0.0012 \\
\hline Vanadium, V & 0.0016 & 0.0001 & 0.0007 & 0.0001 & 0.0012 & 0.0001 & 0.0008 & 0.0001 \\
\hline Chromium, $\mathrm{Cr}$ & 0 & 0.001 & 0 & 0.001 & 0 & 0.001 & 0 & 0.001 \\
\hline Mangane & 0.0179 & 0.0022 & 0.0122 & & 0.0071 & 0.0021 & 0.0053 & 0.0021 \\
\hline Iron, $\mathrm{Fe}$ & 0.7335 & 0.0152 & 0.422 & 0.0091 & 0.426 & 0.0092 & 0.1505 & 0.0043 \\
\hline Cobalt, Co & 0 & 0.0001 & 0 & 0.0001 & 0 & 0.0001 & 0 & 0.0001 \\
\hline Nickel, Ni & 0 & 0.0006 & 0 & 0.0006 & 0 & 0.0006 & 0 & 0.0006 \\
\hline Copper, $\mathrm{Cu}$ & 0.0049 & 0.0009 & 0.0022 & 0.0009 & 0.0062 & 0.0009 & 0.0028 & 0.0009 \\
\hline Zinc, $\mathrm{Zn}$ & 0.004 & 0.0009 & 0.0019 & 0.0009 & 0.003 & 0.0009 & 0.0019 & 0.0009 \\
\hline Gallium, Ga & 0 & 0.0031 & 0 & 0.0031 & 0.0009 & 0.0031 & 0.0017 & 0.0031 \\
\hline Arsenic, As & 0 & 0.0001 & 0 & 0.0001 & 0 & 0.0001 & 0 & 0.0001 \\
\hline Selenium, $\mathrm{Se}$ & 0 & 0.0021 & 0.0007 & 0.0021 & 0.0001 & 0.0021 & 0.0004 & 0.0021 \\
\hline Bromine, $\mathrm{Br}$ & 0.0025 & 0.0015 & 0.0015 & 0.0015 & 0.0026 & 0.0015 & 0.005 & 0.0015 \\
\hline Rubidium, Rh & 0.0029 & 0.0011 & 0.0022 & 0.0011 & 0.0011 & 0.0011 & 0.0006 & 0.0011 \\
\hline Strontium, $\mathrm{Sr}$ & 0.0115 & 0.002 & 0.006 & 0.002 & 0.0041 & 0.002 & 0.0017 & 0.002 \\
\hline Yttrium, Y & 0.0001 & 0.0015 & 0.0008 & 0.0015 & 0.001 & 0.0015 & 0.0008 & 0.0015 \\
\hline Zirconium, $\mathrm{Zr}$ & 0.0035 & 0.0035 & 0 & 0.0034 & 0.0002 & 0.0034 & 0.0006 & 0.0034 \\
\hline Niobium, $\mathrm{Nb}$ & 0 & 0.0026 & 0 & 0.0026 & 0 & 0.0026 & 0 & 0.0026 \\
\hline Molybdenum, Mo & 0 & 0.0024 & 0 & 0.0024 & 0 & 0.0024 & 0 & 0.0024 \\
\hline
\end{tabular}


Table 6. Results of the chemical analysis for selected filters from Crater Flat. Chemical components with concentrations higher than twice the uncertainty are in bold, while those with concentrations lower than twice the uncertainty are in italics. Concentrations are in $\mu \mathrm{g} / \mathrm{m}^{3}$ (continued).

\begin{tabular}{|c|c|c|c|c|c|c|c|c|}
\hline \multirow{3}{*}{$\begin{array}{l}\text { DATE } \\
\text { SIZE }\end{array}$} & \multicolumn{4}{|c|}{$6 / 05 / 2007$} & \multicolumn{4}{|c|}{$7 / 29 / 2007$} \\
\hline & \multicolumn{2}{|c|}{$\mathrm{PM}_{10}$} & \multicolumn{2}{|c|}{$\mathrm{PM}_{2.5}$} & \multicolumn{2}{|c|}{$\mathrm{PM}_{10}$} & \multicolumn{2}{|c|}{$\mathrm{PM}_{2.5}$} \\
\hline & Conc. & Uncer. & Conc. & Uncer. & Conc. & Uncer. & Conc. & Uncer. \\
\hline Palladium, $\mathrm{Pd}$ & 0 & 0.0045 & 0 & 0.0045 & 0.0001 & 0.0045 & 0 & 0.0045 \\
\hline Silver, Ag & 0 & 0.0041 & 0 & 0.0041 & 0 & 0.0041 & 0.0016 & 0.0041 \\
\hline Cadmium, $\mathrm{Cd}$ & 0 & 0.0052 & 0 & 0.0052 & 0 & 0.0052 & 0 & 0.0052 \\
\hline Indium, In & 0.0004 & 0.003 & 0 & 0.003 & 0.0015 & 0.003 & 0 & 0.003 \\
\hline Tin, Sn & 0 & 0.0039 & 0 & 0.0039 & 0.0006 & 0.0039 & 0.0025 & 0.0039 \\
\hline Antimony, Sb & 0 & 0.0073 & 0 & 0.0073 & 0 & 0.0073 & 0 & 0.0073 \\
\hline Cesium, Cs & 0 & 0.0012 & 0 & 0.0012 & 0 & 0.0012 & 0 & 0.0012 \\
\hline Barium, Ba & 0 & 0.0006 & 0 & 0.0006 & 0 & 0.0006 & 0 & 0.0006 \\
\hline Lanthanum, La & 0 & 0.0009 & 0 & 0.0009 & 0 & 0.0009 & 0 & 0.0009 \\
\hline Cerium, $\mathrm{Ce}$ & 0 & 0.0013 & 0 & 0.0013 & 0 & 0.0013 & 0.0007 & 0.0013 \\
\hline Samarium, $\mathrm{Sa}$ & 0 & 0.0018 & 0 & 0.0018 & 0.0009 & 0.0018 & 0 & 0.0018 \\
\hline Europium, Eu & 0.0003 & 0.0064 & 0.0028 & 0.0064 & 0 & 0.0064 & 0 & 0.0064 \\
\hline Terbium, Tb & 0 & 0.0024 & 0 & 0.0024 & 0 & 0.0024 & 0 & 0.0024 \\
\hline Hafnium, Hf & 0 & 0.0139 & 0 & 0.0139 & 0 & 0.0139 & 0 & 0.0139 \\
\hline Tantalum, Ta & 0 & 0.0117 & 0 & 0.0117 & 0 & 0.0117 & 0 & 0.0117 \\
\hline Tungsten, W & 0.0087 & 0.0168 & 0.0018 & 0.0168 & 0.0058 & 0.0168 & 0.0026 & 0.0168 \\
\hline Iridium, Ir & 0 & 0.0036 & 0 & 0.0036 & 0 & 0.0036 & 0 & 0.0036 \\
\hline Gold, Au & 0 & 0.0078 & 0.0001 & 0.0078 & 0 & 0.0078 & 0 & 0.0078 \\
\hline Mercury, Hg & 0 & 0.0024 & 0 & 0.0024 & 0 & 0.0024 & 0 & 0.0024 \\
\hline Thallium, Th & 0 & 0.0025 & 0 & 0.0025 & 0 & 0.0025 & 0 & 0.0025 \\
\hline Lead, $\mathrm{Pb}$ & 0 & 0.0025 & 0.0002 & 0.0025 & 0 & 0.0025 & 0 & 0.0025 \\
\hline Uranium, U & 0 & 0.0041 & 0 & 0.0041 & 0 & 0.0041 & 0.0021 & 0.0041 \\
\hline
\end{tabular}

$\mathrm{OC}=$ organic carbon

$\mathrm{EC}=$ elemental carbon

$\mathrm{OP}=$ optical pyrolysis

$\mathrm{TT}=$ transmittance

With respect to the chemical composition of $\mathrm{PM}_{10}$ and $\mathrm{PM}_{2.5}$, the following patterns were observed:

- $\operatorname{Sulfur}(\mathrm{S})$ was mostly in the form of sulfate $\left(\mathrm{SO}_{4}{ }^{2-}\right)$ with sulfate-to-sulfur ratio of 2.90 to 4.90. Sulfate and ammonium were almost entirely associated with fine particles, while less than 30 percent of nitrate $(23 \%$ to $30 \%)$ was measured in $\mathrm{PM}_{2.5}$. Ammonium-to-sulfate molar ratios varied from 1.55 to 1.93 , suggesting that sulfate aerosols were mostly in the form of ammonium bisulfate, $\left(\mathrm{NH}_{4}\right) \mathrm{HSO}_{4}(\mathrm{Malm}$ et al., 2002). Nitrates appeared to be partially neutralized by ammonium in the fine particle mode, while coarse particles nitrates may be from evaporites in soils or the product of the reactions of nitric acid with soil dust elements such as $\mathrm{Ca}^{2+}, \mathrm{Mg}^{2+}, \mathrm{Na}^{+}$, and $\mathrm{K}^{+}$ (Lefer and Talbot, 2001).

- Carbonaceous aerosol was predominantly in fine particles. For $\mathrm{PM}_{2.5}$, organic carbon (OC) concentrations accounted for 23 to 46 percent of particle mass, while very low 
$\mathrm{EC} / \mathrm{OC}$ ratios were indicative of the absence of high temperature combustion-related sources.

- Soluble potassium $\left(\mathrm{K}^{+}\right)$accounted for 20 to 66 percent of total potassium in $\mathrm{PM}_{10}$ and for more than 50 percent of total potassium in $\mathrm{PM}_{2.5}$. Soluble potassium in fine particles is a tracer of biomass burning, which suggested the significant impact of emissions from local and/or regional fire (prescribed or wildfire) events. On the other hand, salts in desert soil may be the source of soluble potassium that is mostly present in the coarse fraction. This was further supported by the estimates of nonsoil potassium $\mathrm{K}_{\text {non-soil }}\left(\mathrm{K}_{\text {total- }}(0.26 \mathrm{x}[\mathrm{Al}])\right)$ that were comparable to measured watersoluble $\mathrm{K}^{+}$. Water soluble $\mathrm{K}^{+}$is also present as salts in soils.

- $\quad$ Ratios of $\mathrm{Al} / \mathrm{Si}(0.37$ to 0.40$) \mathrm{K} / \mathrm{Fe}(0.51$ to 0.80$)$ were comparable to those determined for samples collected at the Interagency Monitoring of Protected Visibility Environments (IMPROVE) sites in the western United States (Al/Si: 0.31 to $0.43, \mathrm{~K} / \mathrm{Fe}: 0.67$ to $0.78, \mathrm{Al} / \mathrm{Ca}: 1.4$ to 1.7$)$ when soil dust was the major component of particulate matter (Kavouras et al., 2005).

The IMPROVE mass calculation was applied to reconstruct aerosol mass into five major species, namely sulfate, nitrate, organic, light-absorbing carbon, and soil. For this scheme, sulfate and nitrate are assumed to be in the forms of ammonium sulfate $\left[\left(\mathrm{NH}_{4}\right)_{2} \mathrm{SO}_{4}\right]$ and ammonium nitrate $\left[\mathrm{NH}_{4} \mathrm{NO}_{3}\right]$, respectively (Malm et al., 2004). Organic mass concentration $[\mathrm{OMC}]$ was estimated as $[\mathrm{OMC}]=1.4 \mathrm{x}[\mathrm{OC}]$, where $[\mathrm{OC}]$ is the organic carbon concentration. The 1.4 factor was used to estimate for elements not measured (mainly hydrogen and oxygen) in organic compounds (White and Roberts, 1977). Soil mass concentration [SOIL] was estimated as the sum of the elements present in the soil as oxides calculated as follows:

$[\mathrm{SOIL}]=2.2 \times[\mathrm{Al}]+2.49[\mathrm{Si}]+1.63 \times[\mathrm{Ca}]+2.42 \times[\mathrm{Fe}]+1.94 \times[\mathrm{Ti}]$. Therefore, the reconstructed aerosol mass was estimated as follows:

$[$ Aerosol Mass $]=(128 / 96) \times\left[\mathrm{SO}_{4}\right]+(80 / 62) \times\left[\mathrm{NO}_{3}\right]+\mathrm{EC}+[\mathrm{OMC}]+[\mathrm{SOIL}]$

Figure 6 shows the reconstructed concentrations of ammonium sulfate, ammonium nitrate, organic carbon, elemental carbon, and soil for $\mathrm{PM}_{10}$ and $\mathrm{PM}_{2.5}$ collected on June 5, 2007 and July 29, 2007 in Crater Flat. Considering the positive bias for organic carbon measurements:

- $\quad$ Reconstructed particle mass accounted for 83 to 95 percent of measured $\mathrm{PM}_{10}$ mass and for 101 to 119 percent of $\mathrm{PM}_{2.5}$ mass.

- Carbonaceous aerosol (OMC and EC) appeared to account for 22 to 36 percent of $\mathrm{PM}_{10}$ and 32 to 63 percent of $\mathrm{PM}_{2.5}$.

- Soil represented 37 to 49 percent of $\mathrm{PM}_{10}$ and about 29 to 56 percent of $\mathrm{PM}_{2.5}$ mass, while sulfate contributed between 8 and 15 percent on $\mathrm{PM}_{10}$ and 11 to 22 percent on $\mathrm{PM}_{2.5}$ (Figure 6).

The differences of $\mathrm{PM}_{10}$ and $\mathrm{PM}_{2.5}$ fractions are due to higher concentration of soil elements in the coarse fraction (particles with diameter between 2.5 and $10 \mu \mathrm{m}$ ). Higher $\mathrm{PM}_{2.5}$ mass concentrations for July 29, 2007, may be attributed to increased concentrations of organic carbon and to a lesser extent on soil and sulfate concentrations. 


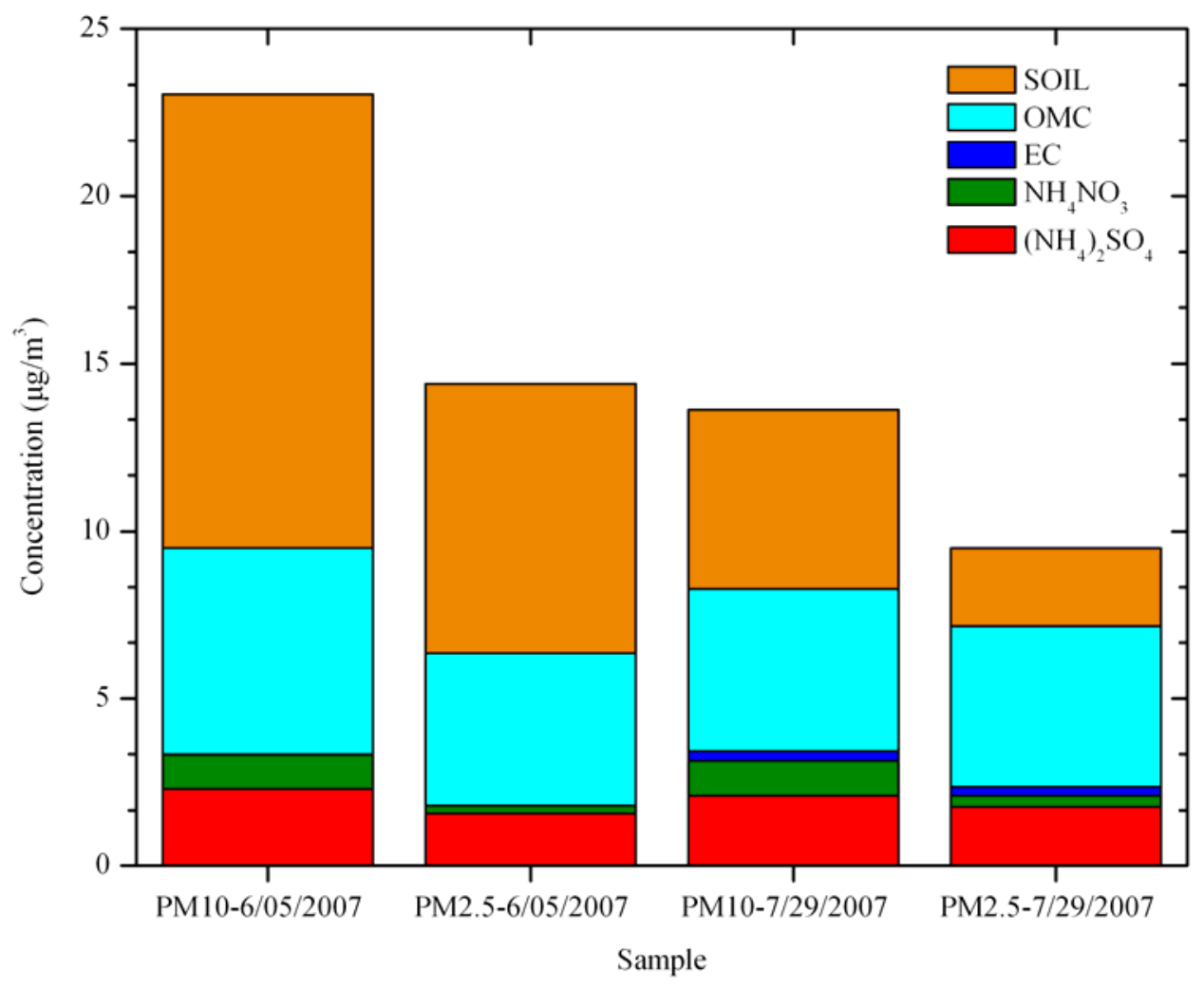

Figure 6. Reconstructed mass for PM10 and PM2.5 based on chemical composition.

\section{Aerosol Monitoring}

\section{Monitor Description and Procedures}

The DUSTTRAK ${ }^{\mathrm{TM}}$ Aerosol Monitors from TSI continuously measured $\mathrm{PM}_{10}$ and $\mathrm{PM}_{2.5}$ mass concentrations (Figure 7). They are portable, battery operated laser photometers that provide measurements of particle mass, based on light scattering. Atmospheric aerosol passes through a size selective inlet (either $\mathrm{PM}_{10}$ or $\mathrm{PM}_{2.5}$ ) and is directed to an optics chamber at a flow rate of $1.7 \mathrm{l} / \mathrm{min}$. The light source is a laser diode that emits light at a wavelength of $780 \mathrm{~nm}$. Sampled aerosol is drawn into the sensing chamber where it is illuminated with a narrow beam of laser light. Light scattered by aerosol particles is collected by a set of lenses and focused onto the photodetector. The detector signal is proportional to the amount of scattered light, which is proportional to the mass concentration of the aerosol. Voltage is read by the processor and multiplied by an internal calibration constant to yield mass concentration. The calibration constant is pre-set by the manufacturer for scattering characteristics of the respirable mass of ISO 12103-1, Al test dust. Local variations in aerosol particle size distribution and composition relative to this standard may result in differences in the actual response factor of the instrument. The operation, calibration, and maintenance of the DUSTRAK is described in DRI SOP 1.211-2 "TSI INCORPORATED MODEL 8520 DUSTTRAK AEROSOL MONITOR FOR THE YUCCA MOUNTAIN AIR QUALITY PROGRAM.” 


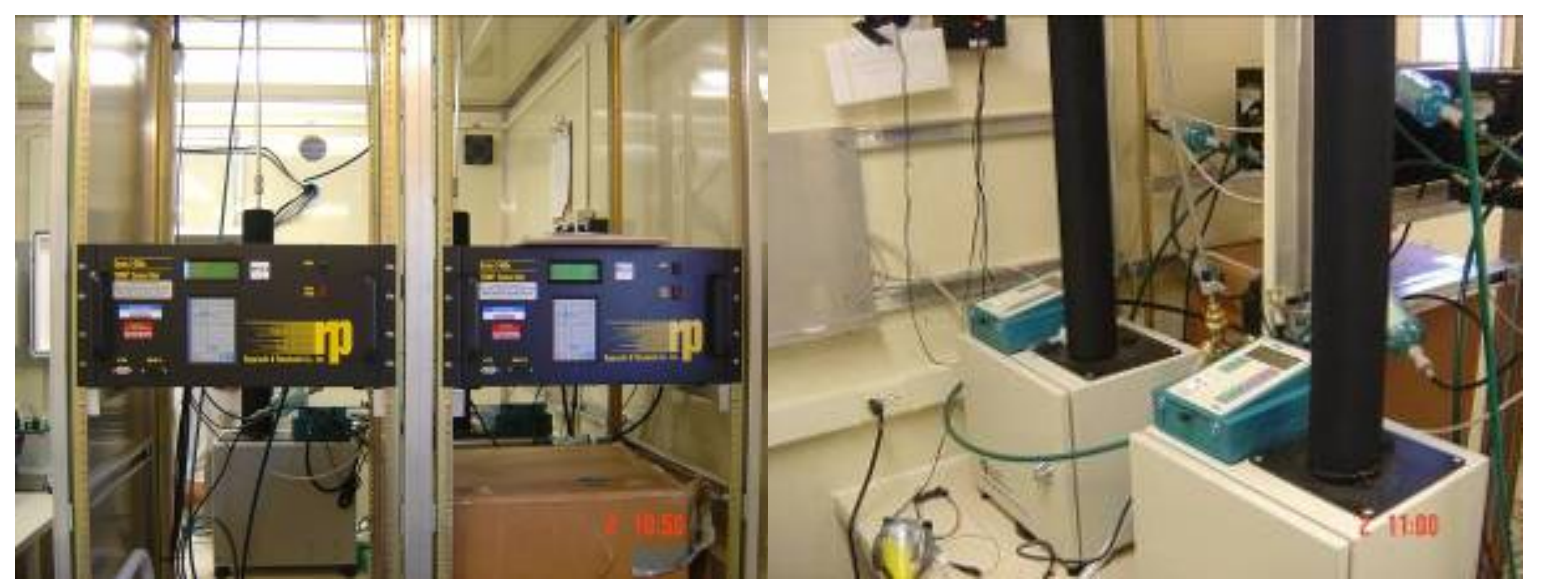

Figure 7. Left photograph: The front panels of $\mathrm{PM}_{10}$ (right on the left photograph) and $\mathrm{PM}_{2.5}$ (left on the left photograph) of TEOM. Right photograph: The DUSTTRAK monitors (green) resting on top of the two TEOM measuring units.

Both $\mathrm{PM}_{10}$ and $\mathrm{PM}_{2.5}$ DUSTTRAK inlets were attached on a wide "Y" connector, which was connected to one leg of a second "Y" (Figure 8). A funnel with a suction fan was connected to the other leg of the second "Y" to achieve fast exchange of ambient air into the sampling line. Flow calibration and zero-test were performed on the day of installation (May $25,2007)$ and subsequent site visits. Deviations in flow were predominantly due to failure of the pump diaphragm. In those cases, the instrument was replaced. Deviations of the zero check were corrected by performing zero calibration according to the manufacturer's operational instruction manual.

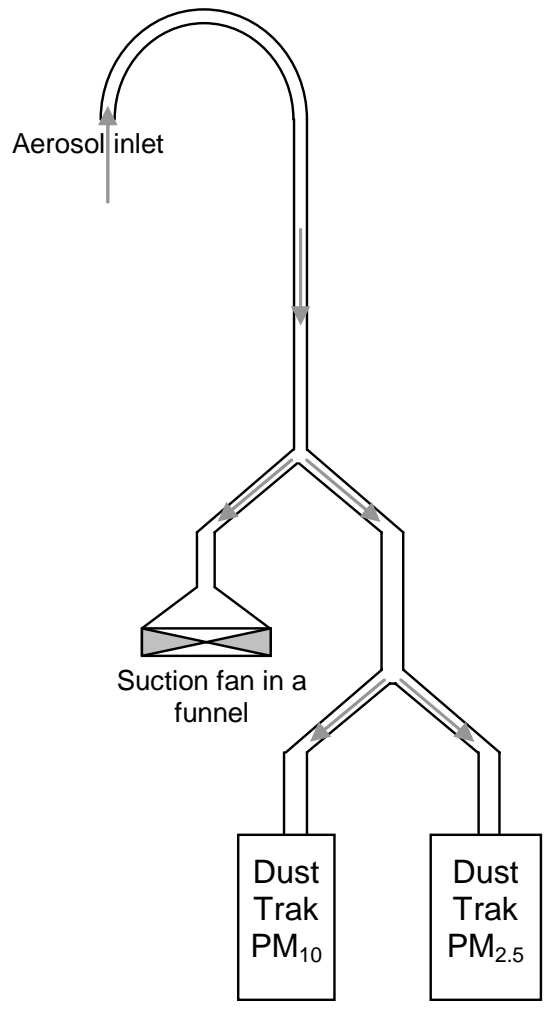


Figure 8. Schematic drawing of the sampling inlet for the DUSTTRAK (not to scale).

\section{Continuous DUSTTRAK Measurements of PM$\underline{10} \underline{\text { and PM }} 2 \underline{2.5}$}

Trends and correlations of particle mass are examined using hourly DustTtak data integrated for 24 hours (from 0:00. to 23:59). Statistics of 24-h particle mass are presented in Table 4.

Table 4. Statistics for 24-h $\mathrm{PM}_{10}$ and $\mathrm{PM}_{2.5}$ mass concentrations.

\begin{tabular}{rrrrrr}
\hline & \multicolumn{1}{c}{ Mean } & Median & Minimum & Maximum & Std. Deviation \\
\hline $\mathrm{PM}_{10}$ & 102.84 & 70.92 & 0.30 & $1,001.00$ & 156.09 \\
$\mathrm{PM}_{2.5}$ & 24.01 & 10.58 & 0.05 & 541.60 & 32.50 \\
\hline
\end{tabular}

Twenty-four-hour $\mathrm{PM}_{10}$ levels ranged from 0.30 to $1,001.0 \mu \mathrm{g} / \mathrm{m}^{3}$, with a mean of $102.84(\mathrm{~s}=156.09) \mu \mathrm{g} / \mathrm{m}^{3}$, while $\mathrm{PM}_{2.5}$ concentrations varied from 0.05 to $541.60 \mu \mathrm{g} / \mathrm{m}^{3}$, with a mean of $24.1(\mathrm{~s}=32.50) \mu \mathrm{g} / \mathrm{m}^{3} . \mathrm{PM}_{10}$ mass concentrations increased drastically after July 12,2007 . It should be mentioned that the DUSTTRAK (S/N: 85200794) was replaced by another (S/N: 85200795) on July 13, 2007. Given the limitations of DUSTTRAK to measure coarse particles, the intercomparison between filter samples and DUSTTRAK will determine whether variations were caused by atmospheric conditions or not (Figure 9). A consistent relationship between PM fractions was observed before July 12, 2007, with fine particles being accounted for about three quarters of $\mathrm{PM}_{10}\left(\mathrm{PM}_{2.5} / \mathrm{PM}_{10}\right.$ ratio of 0.75$)$ and about 10 percent for the remaining period (Figure 10). All the operating parameters (flow and zero) were within the acceptable ranges (flow: $1.7 \pm 0.1$ liters/min and Zero: $0.000 \pm 0.001$ $\mathrm{mg} / \mathrm{m}^{3}$ ). While differences in particle mass for weekdays/weekends were not statistically significant, somewhat higher $\mathrm{PM}_{10}$ levels were measured on Monday (Day\#1) and Tuesday (Day \#2) (Figure 11).

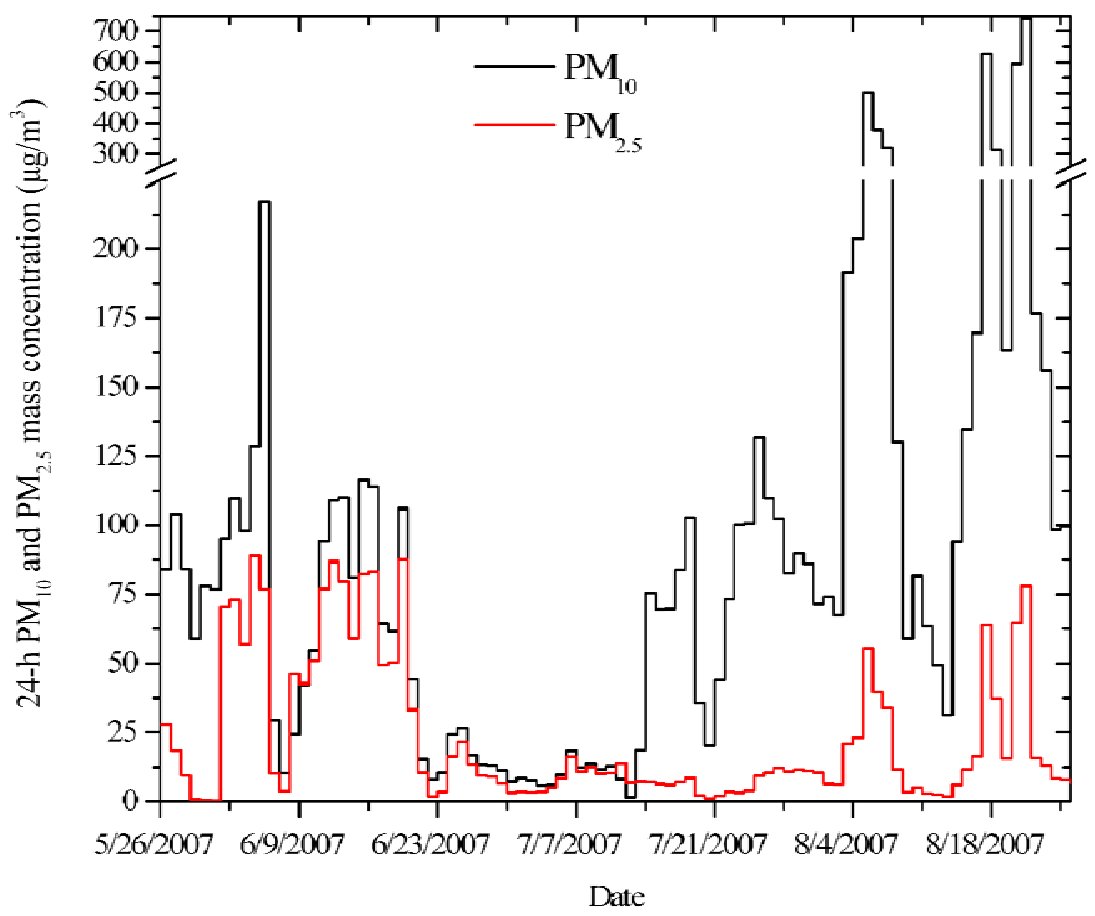


Figure 9. Mean 24-h $\mathrm{PM}_{10}$ and $\mathrm{PM}_{2.5}$ mass concentrations measured by DUSTTRAK at Site \#7 (Crater Flat).

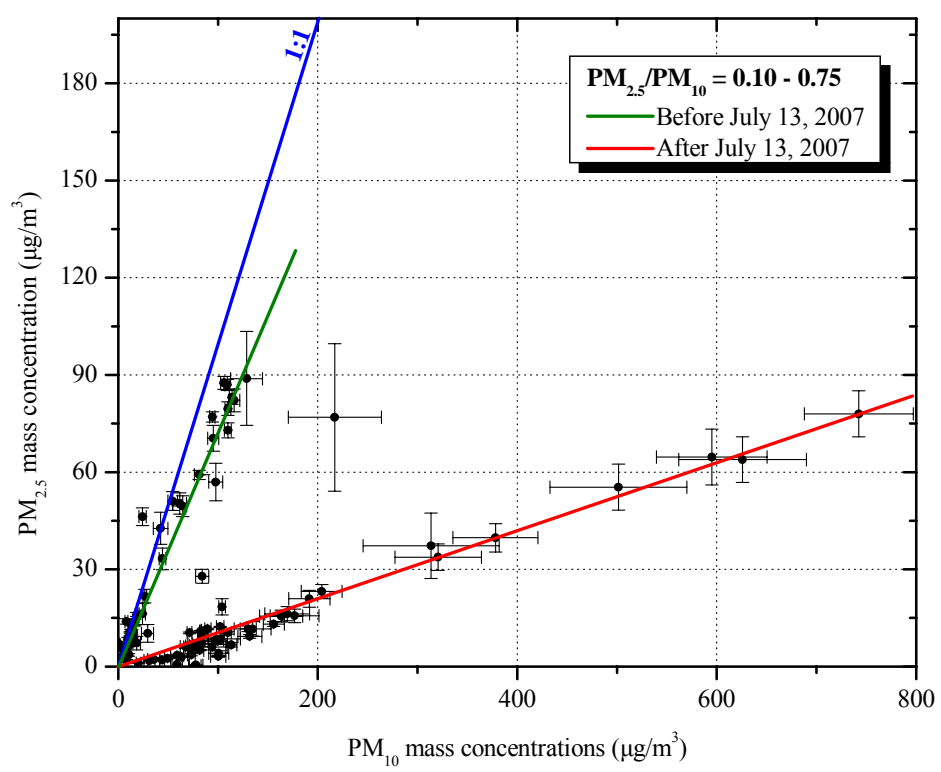

Figure 10. $\mathrm{PM}_{2.5} / \mathrm{PM}_{10}$ mass ratios at Site \#7 (Crater Flat) Error bars represent the standard error of the mean.

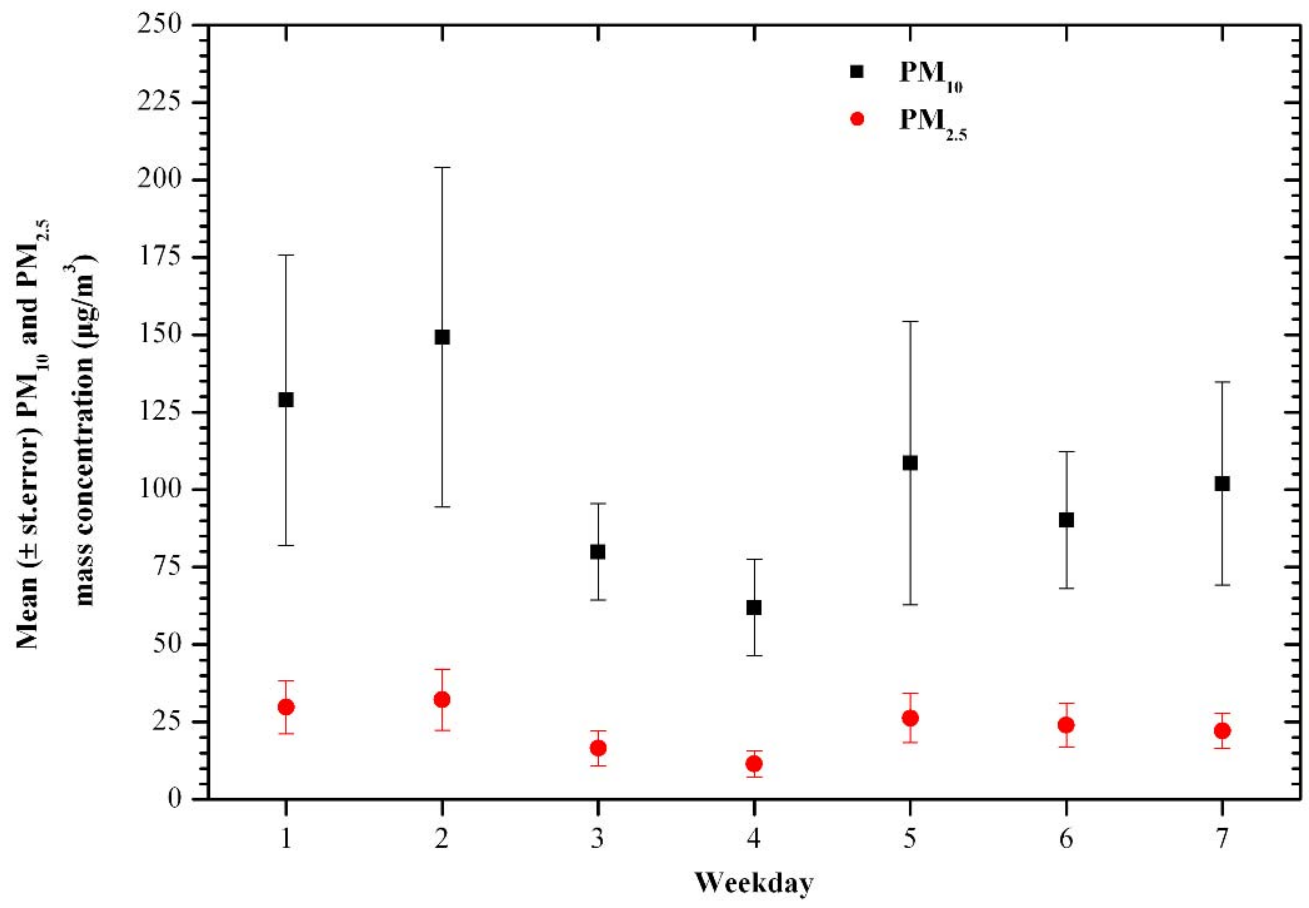

Figure 11. Variation of mean ( \pm st.error) $\mathrm{PM}_{10}$ and $\mathrm{PM}_{2.5}\left(\mu \mathrm{g} / \mathrm{m}^{3}\right)$ in weekdays and weekends at Site \#7 (Crater Flat) (Monday=1, Tuesday $=2$, Wednesday $=3$, Thursday $=4$, Friday $=5$, Saturday $=6$, Sunday $=7$ ). 


\section{Comparison of Filter to Continuous Results}

Figure 12 and Figure 13 show the relationships between $\mathrm{PM}_{10}$ and $\mathrm{PM}_{2.5}$ measured by DUSTTRAK and filter-based methods. The correlations between PM measurements by DUSTTRAK and filter methods were poor, with correlation coefficients of 0.52 for $\mathrm{PM}_{10}$ and 0.206 for $\mathrm{PM}_{2.5}$. The slopes for $\mathrm{PM}_{10}$ and $\mathrm{PM}_{2.5}$ measured by DUSTTRAK were 7.46505 and 1.99644 , respectively, while high intercepts are computed. The poor correlation for $\mathrm{PM}_{10}$ may be attributed to the low range of filter-based values (10 to $\left.20 \mu \mathrm{g} / \mathrm{m}^{3}\right)$.

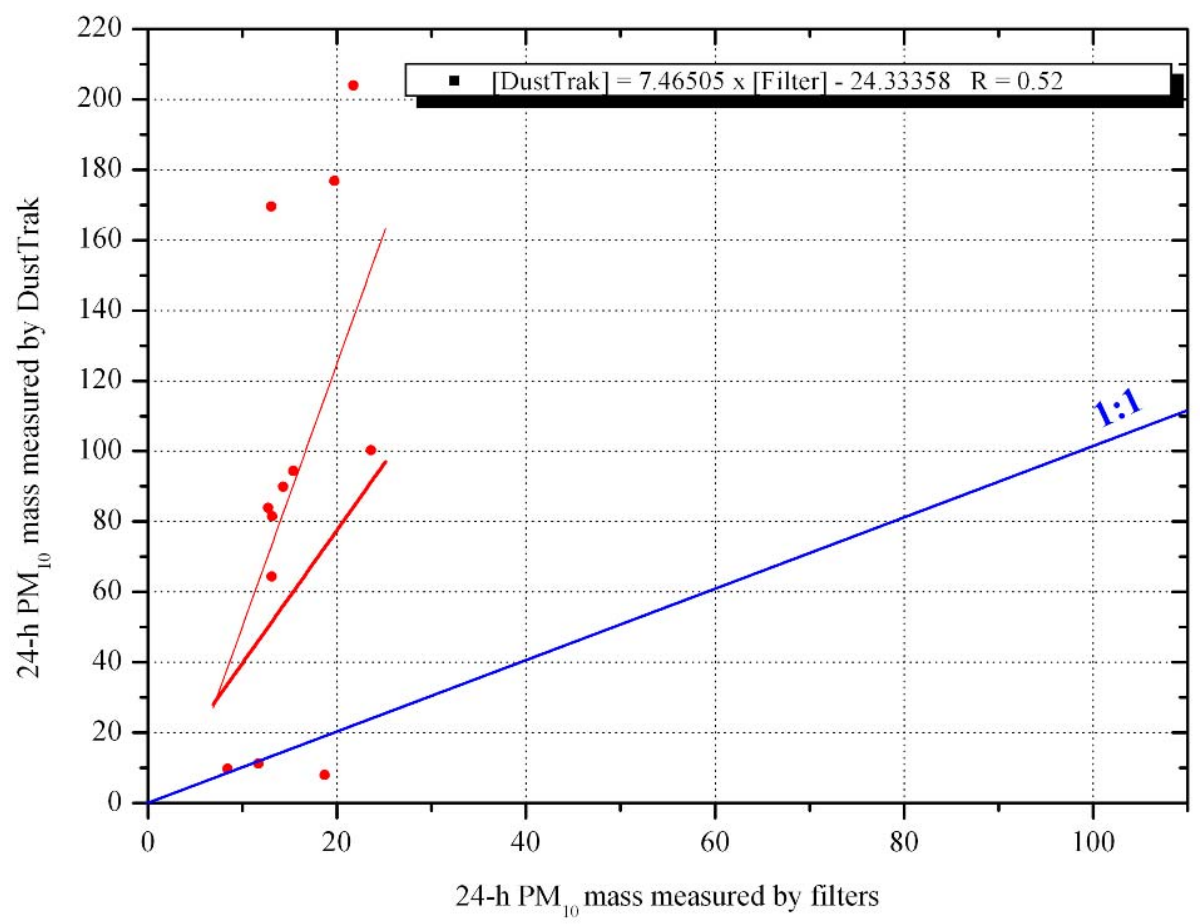

Figure 12. Relationships between $\mathrm{PM}_{10}$ concentrations $\left(\mu \mathrm{g} / \mathrm{m}^{3}\right)$ measured by DUSTTRAK, and filterbased methods. 


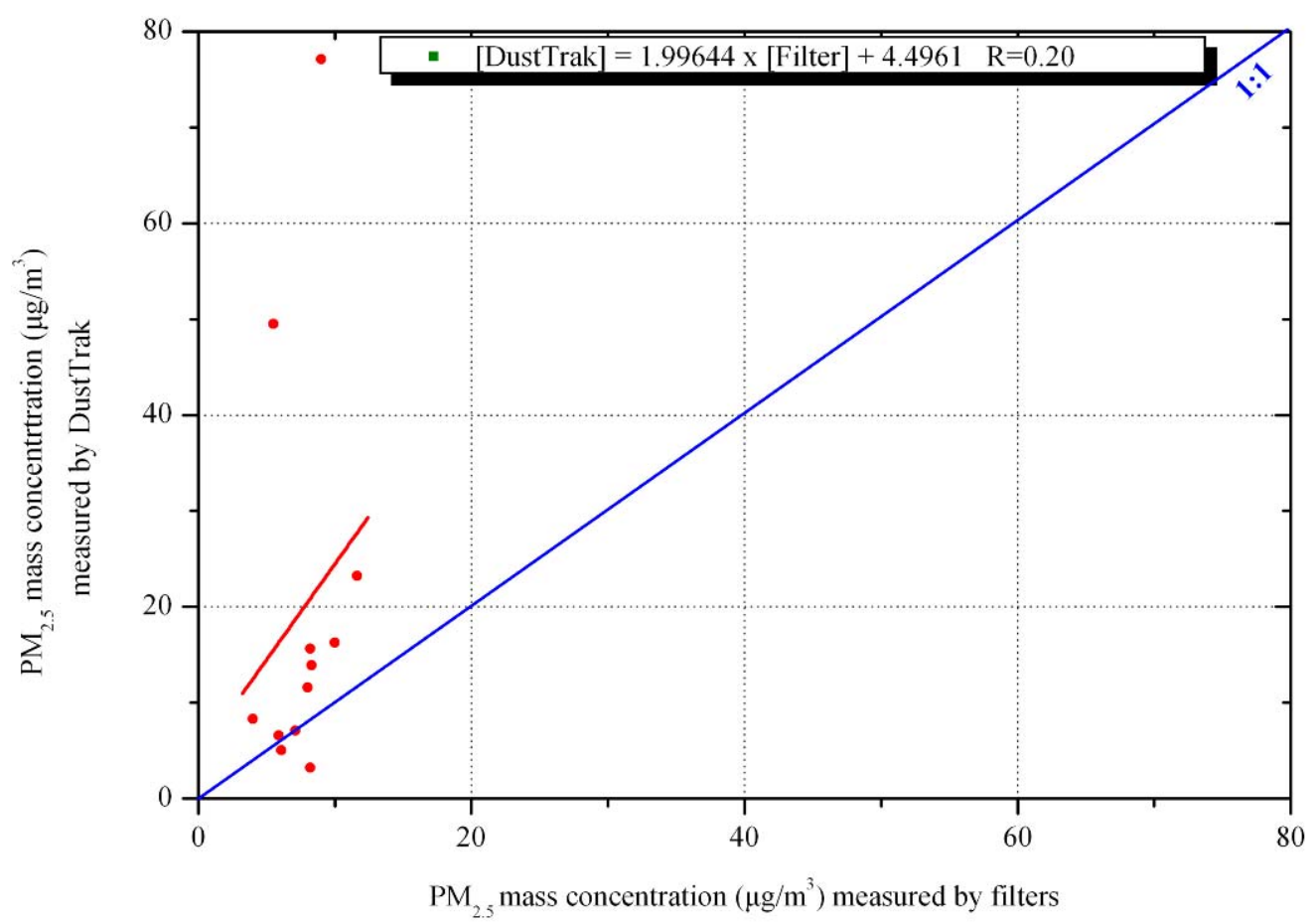

Figure 13. Relationships between $\mathrm{PM}_{2.5}$ concentrations $\left(\mu \mathrm{g} / \mathrm{m}^{3}\right)$ measured by DUSTTRAK, and filterbased methods.

\section{METEOROLOGY}

Variations of hourly data for each meteorological parameter are presented in Figure 14 through 17. Descriptive statistics of hourly data also are presented in Table 5. Solar radiation progressively increased up to 87.4 watts $/ \mathrm{m}^{2}$ (Figure 14). Ambient temperature varied from 50.2 to $109.5^{\circ} \mathrm{F}$ with a mean temperature of $86.0^{\circ} \mathrm{F}$ for the monitoring period (Table 5; Figure 15). Relative humidity remained lower than 60 percent. No rainfall events were recorded.

Table 5. Descriptive statistics of 1-hour meteorological data.

\begin{tabular}{lrcc}
\hline & Mean & Minimum & Maximum \\
\hline Solar radiation $\left(\right.$ watts $\left./ \mathrm{m}^{2}\right)$ & 28.0 & 0.0 & 87.4 \\
Wind speed (miles $/ \mathrm{h})$ & 9.4 & 0.0 & 33.1 \\
Temperature $\left({ }^{\circ} \mathrm{F}\right)$ & 86.0 & 50.2 & 109.5 \\
Relative humidity $(\%)$ & 14.5 & 2.9 & 58.2 \\
\hline
\end{tabular}




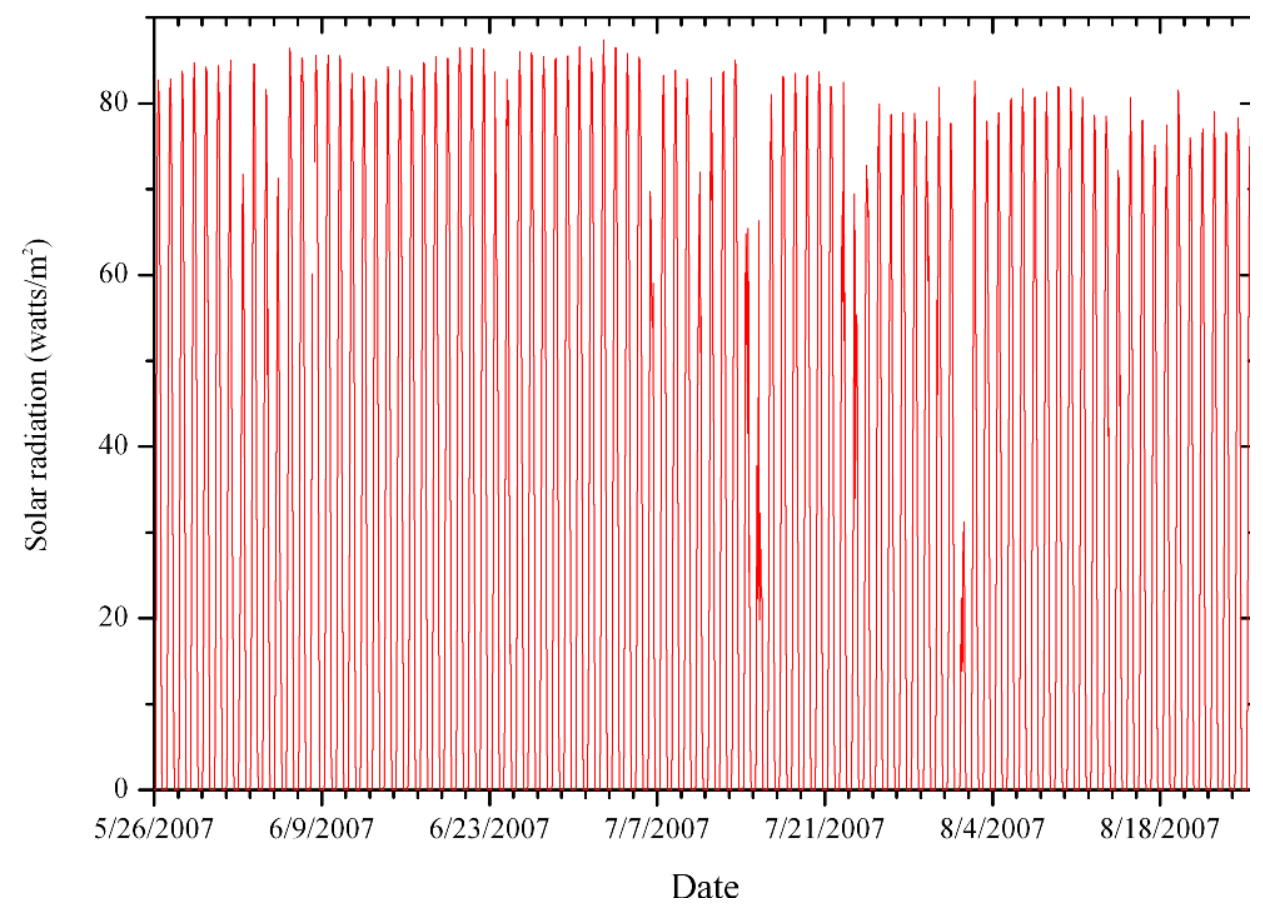

Figure 14. Solar radiation (in watts $/ \mathrm{m}^{2}$ ) at Site \#7 (Crater Flat).

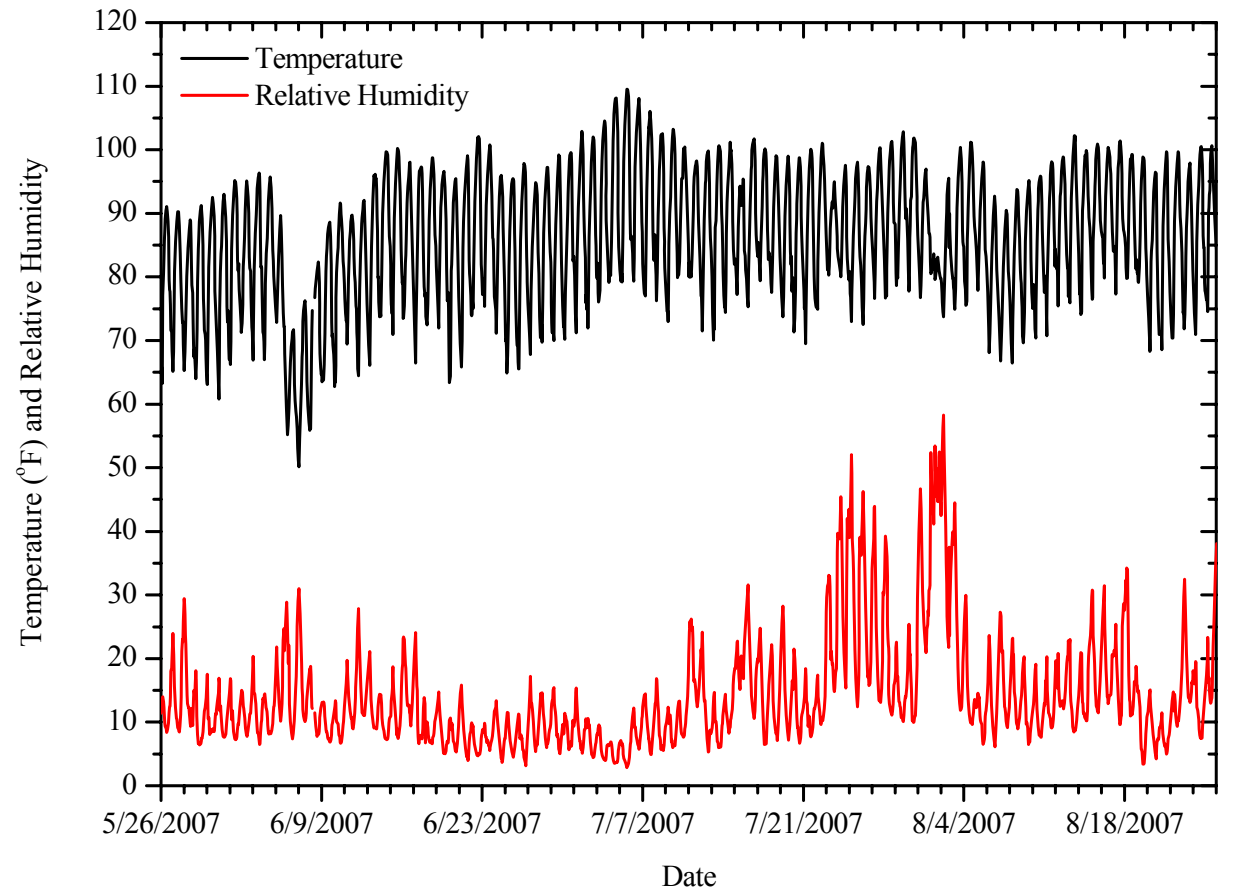

Figure 15 . Temperature (in ${ }^{\circ} \mathrm{F}$ ) and relative humidity at Site \#7 (Crater Flat). 


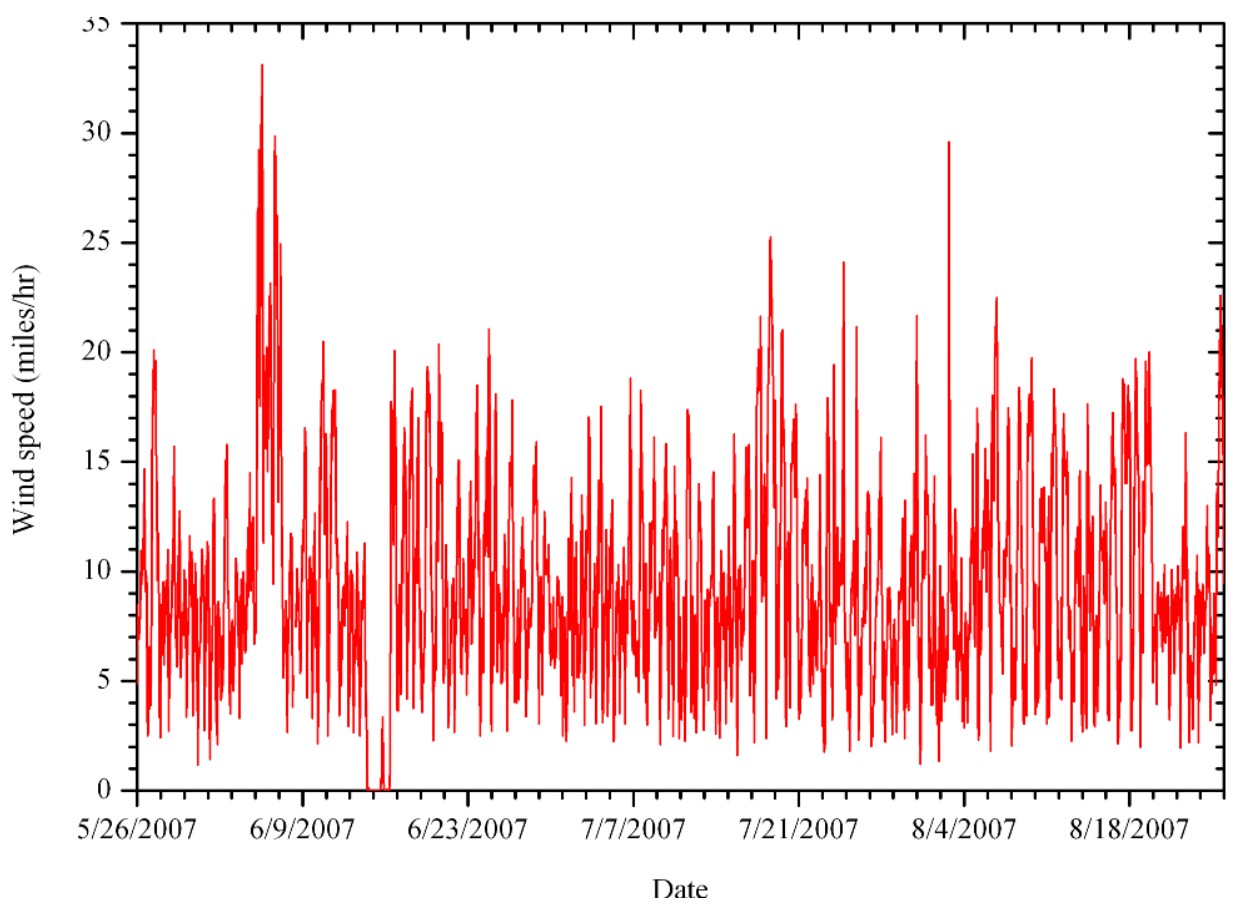

Figure 16. Wind speed (in miles/hr) at Site \#7 (Crater Flat).

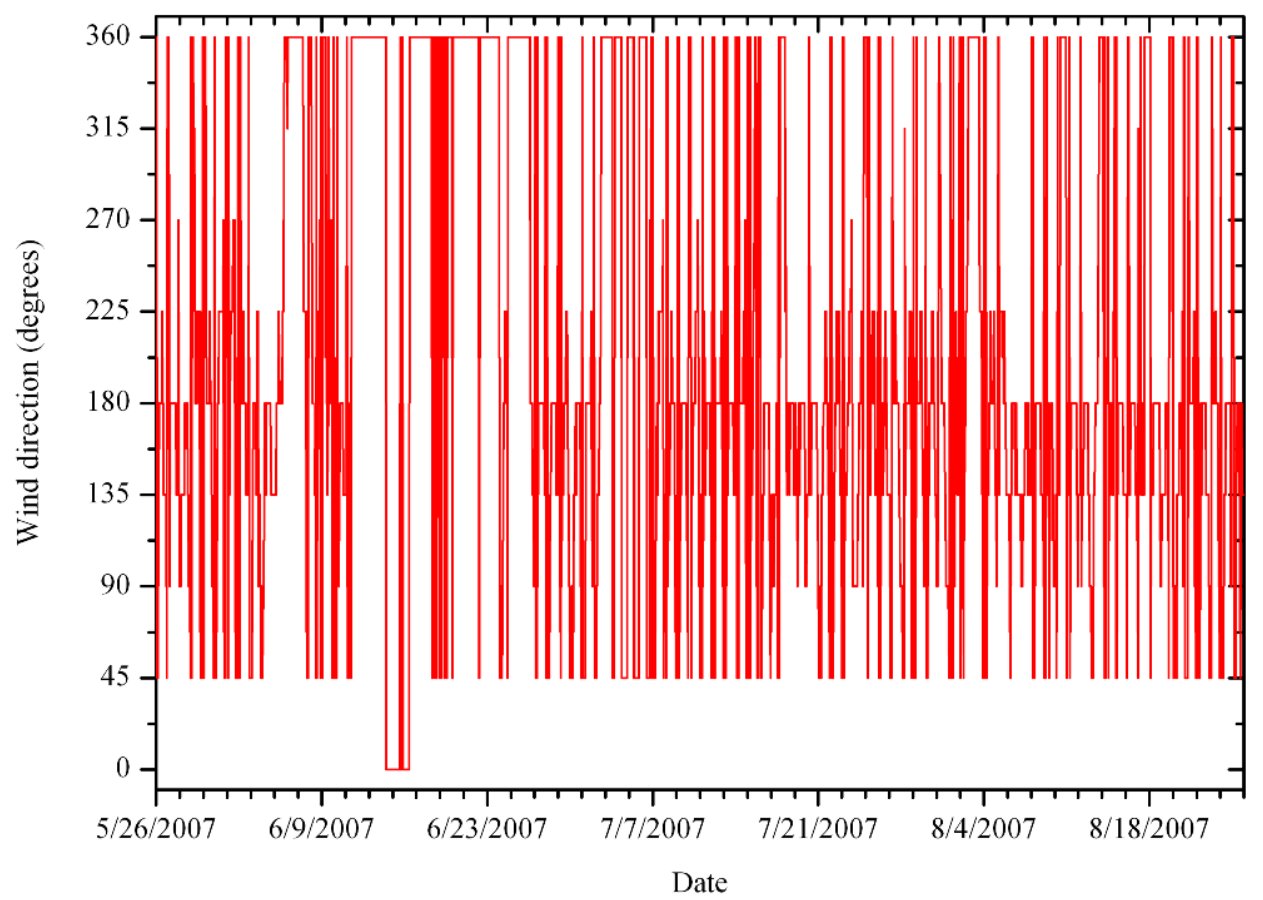

Figure 17. Wind direction at Site \#7 (Crater Flat).

Wind conditions for the monitoring period were described by northerly winds during the night and southerly winds during the day, with wind speeds mostly in the range of 5 to 15 miles/hour (Figure 16 and Figure 17). The classification of wind conditions (Table 6) was 
retrieved from the Federal Meteorological Handbook. The mean wind speed for each direction bin ( 8 bins) is presented in Figure 18.

Table 6. Wind condition classifications.

\begin{tabular}{cl}
\hline Miles/hour & Specification \\
\hline$<1$ & Calm; smoke rises vertically. \\
1 to 5 & Direction of wind shown by smoke drift not by wind vanes. Wind felt on face; \\
& leaves rustle; vanes moved by wind. \\
5 to 9 & Leaves and small twigs in constant motion; wind extends light flag. \\
9 to 14 & Raises dust, loose paper; small branches moved. \\
14 to 23 & Small trees in leaf begin to sway; crested wavelets form on inland waters. Large \\
& $\begin{array}{l}\text { branches in motion; whistling heard in overhead wires; umbrellas used with } \\
\text { difficulty. }\end{array}$ \\
23 to 35 & Whole trees in motion; inconvenience felt walking against wind. Breaks twigs off \\
& trees; impedes progress. \\
35 to 48 & Slight structural damage occurs. Trees uprooted; considerable damage occurs. \\
$>48$ & Widespread damage. \\
\hline (retrieved from Federal Meteorological Handbook; Chapter 5. Wind; \\
http://www.nws.noaa.qov/oso/oso1/oso12/fmh1/fmh1ch5.htm\#chp5link)
\end{tabular}

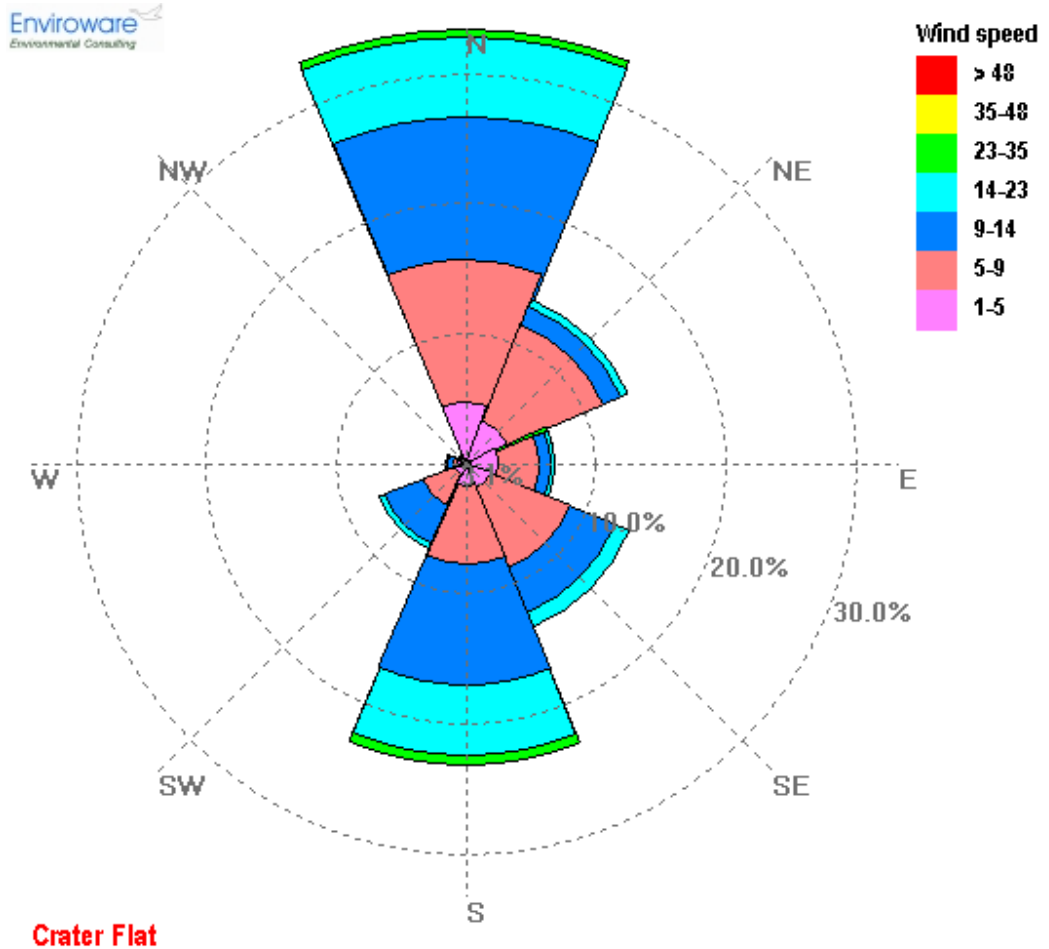

Figure 18. Wind direction and speed at Crater Flat. 
On average, winds blowing from south were slightly stronger (11.5 miles/hr) than those from the north $(9.6 \mathrm{miles} / \mathrm{hr})$. This is controlled by the north-south-trending topography of the region. Lower wind speeds are recorded for winds blowing from the northwest (Figure 19).

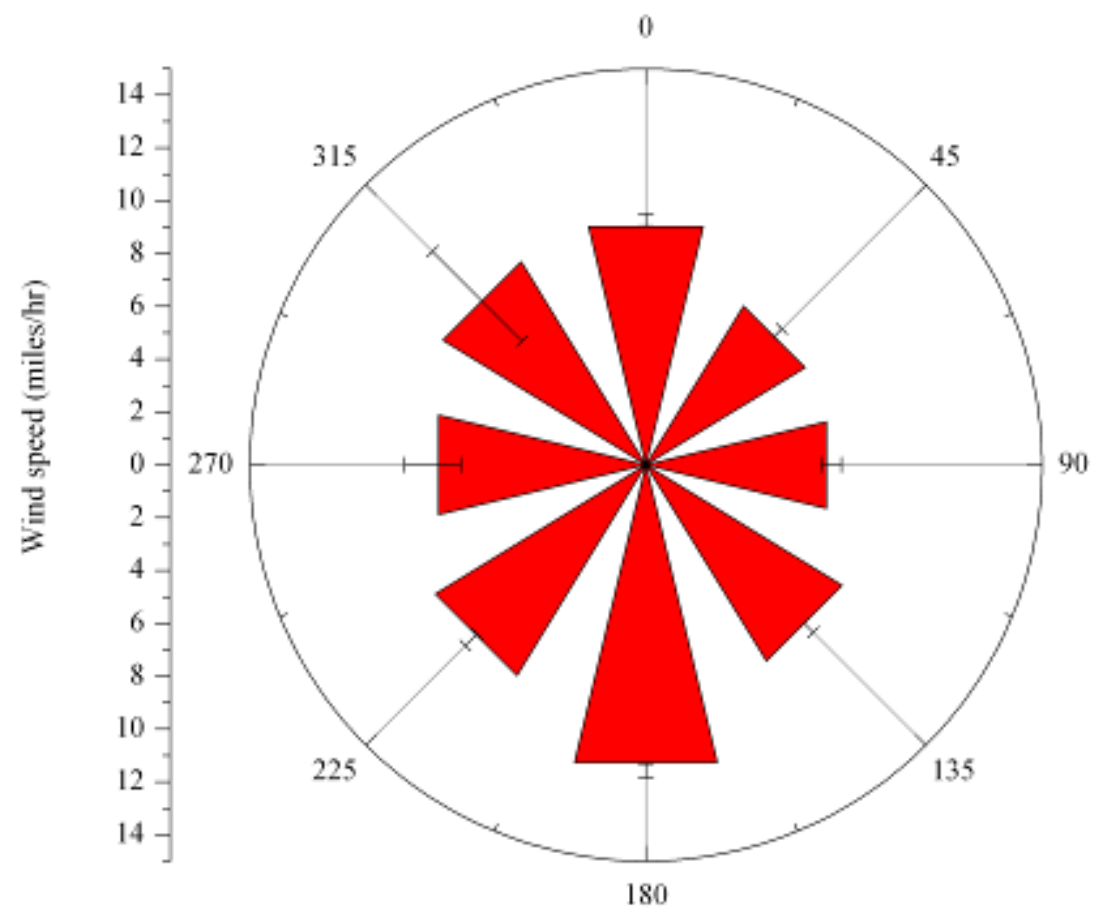

Figure 19. Average wind speed for each wind direction sector. Error bars represent the standard error of the mean.

\section{Relationships of Meteorology with Aerosol Measurements}

Trends and correlations of PM mass with meteorological conditions are shown for hourly DUSTTRAK data. A weak bimodal pattern is observed for both fractions of particle mass (Figure 20). The first mode is associated with comparatively higher particle mass concentration in early morning (7:00 to 9:00 for $\mathrm{PM}_{10}$ and 10:00 to 11:00 for $\mathrm{PM}_{2.5}$ ) followed by a gradual decrease. The second mode for $\mathrm{PM}_{10}$ can be observed in early afternoon (13:00 to 15:00) as winds were increasing, followed by an increase in the evening (19:00 to 22:00). There are significant differences of $\mathrm{PM}_{10}$ and $\mathrm{PM}_{2.5}$ concentrations for different wind directions, with highest $\mathrm{PM}_{10}$ levels for northwest winds, and highest $\mathrm{PM}_{2.5}$ levels for northerly winds (Figure 21 and Figure 22). 


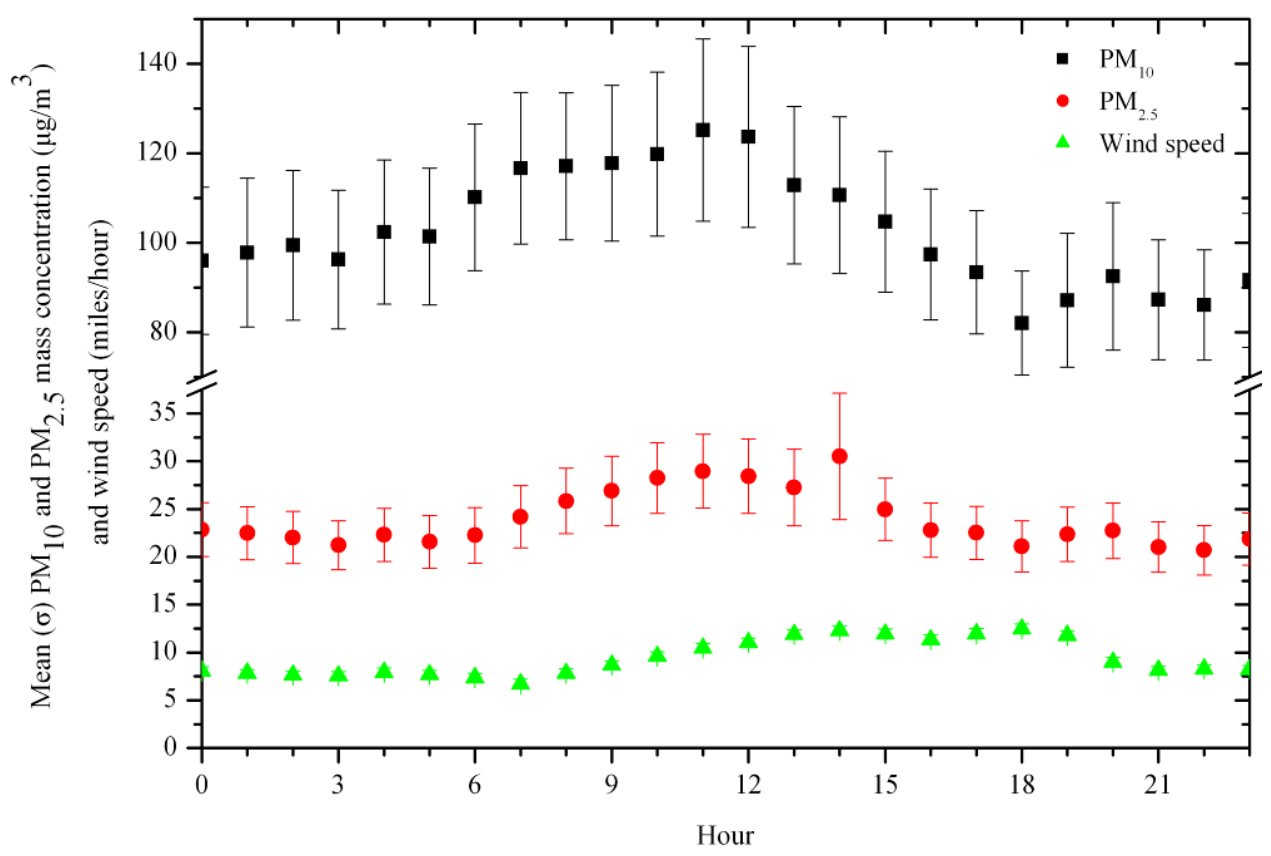

Figure 20. Hourly variation of $\mathrm{PM}_{10}$ and $\mathrm{PM}_{2.5}$ mass concentrations $\left(\mu \mathrm{g} / \mathrm{m}^{3}\right)$ as well as wind speed (miles/hour) at Site \#7 (Crater Flat). Error bars represent the standard error of the mean.

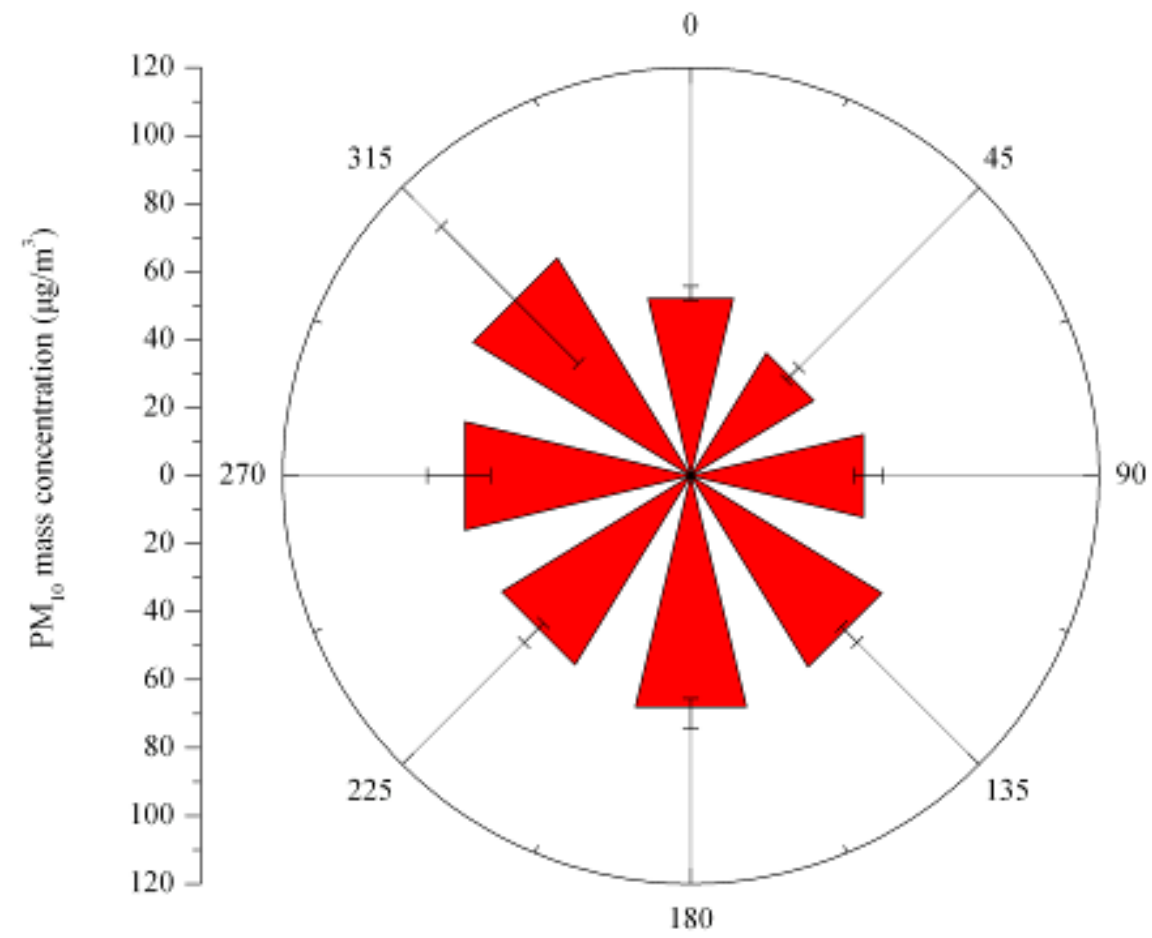

Figure 21. Mean ( \pm st.error) of $\mathrm{PM}_{10}$ mass concentrations $\left(\mu \mathrm{g} / \mathrm{m}^{3}\right)$ for different wind direction sectors at Site \#7 (Crater Flat). 


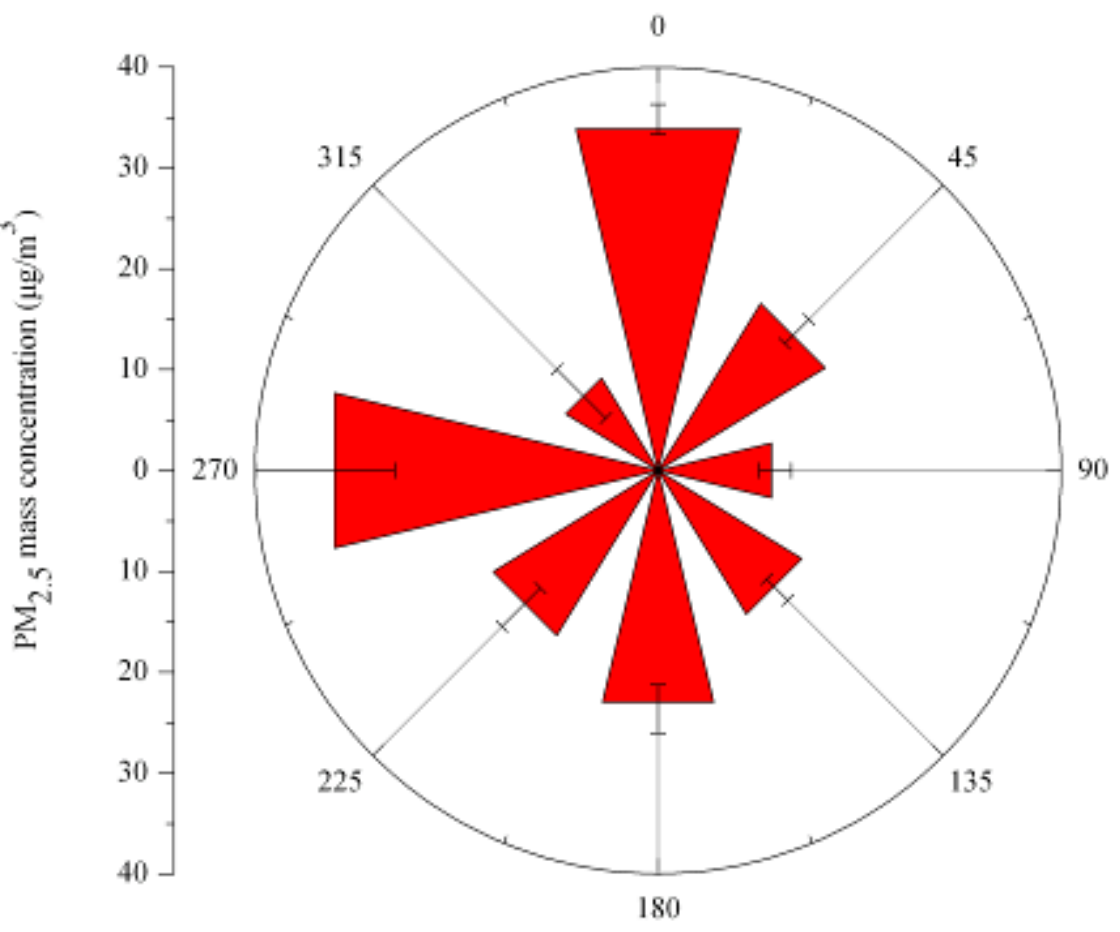

Figure 22. Mean ( \pm st.error) of $\mathrm{PM}_{2.5}$ mass concentrations $\left(\mu \mathrm{g} / \mathrm{m}^{3}\right)$ for different wind direction sectors at Site \#7 (Crater Flat).

\section{CONCLUSIONS}

$\mathrm{PM}_{10}$ and $\mathrm{PM}_{2.5}$ mass concentrations and meteorological conditions were continuously monitored in Crater Flat, from May 25 to August 29, 2007. Because of the remote location of the site, and reliance only on 12-volt batteries and solar power, continuous measurements of $\mathrm{PM}_{10}$ and $\mathrm{PM}_{2.5}$ were obtained using only DUSTTRAKs, not TEOMs. At the same time, integrated samples of $\mathrm{PM}_{10}$ and $\mathrm{PM}_{2.5}$ were collected using FRM samplers on a 1-to-6-day schedule. Two sets of filters (June 05 and July 29, 2009) were analyzed for major anions (sulfate, nitrate, chloride) and cations (sodium and potassium), 40 elements (from sodium to uranium), and elemental and organic carbon. The comparison of $\mathrm{PM}_{10}$ and $\mathrm{PM}_{2.5}$ mass concentrations obtained by continuous monitors and filters showed that differences are associated with the limitations of the operating principle. For example, while light scattering (the measurement technique for DUSTTRAK) is not influenced by volatilization losses and is accurate for fine particles, it performs poorly for coarse particles, resulting in underestimation of $\mathrm{PM}_{10}$ mass. $\mathrm{PM}_{2.5}$ mass measurements obtained by DUSTTRAK, and filter-based methods were also compared.

Mean 24-h concentrations (measured by DUSTTRAK) of $\mathrm{PM}_{10}$ and $\mathrm{PM}_{2.5}$ mass were 58.4 and $24.1 \mu \mathrm{g} / \mathrm{m}^{3}$, which are lower than the 24-h and annual NAAQS standards (24-h $\mathrm{PM}_{10}: 150 \mu \mathrm{g} / \mathrm{m}^{3}, 24-\mathrm{h} \mathrm{PM}_{2.5}: 35 \mu \mathrm{g} / \mathrm{m}^{3}$ ). Particle mass measured on filters varied from 1.5 to $217.1 \mu \mathrm{g} / \mathrm{m}^{3}$ for $\mathrm{PM}_{10}$ and from 0.2 to $88.9 \mu \mathrm{g} / \mathrm{m}^{3}$ for $\mathrm{PM}_{2.5}$. Substantially higher $\mathrm{PM}_{10}$ and $\mathrm{PM}_{2.5}$ levels were associated with increased wind speeds blowing mostly from the north and south. The chemical composition of both $\mathrm{PM}_{10}$ and $\mathrm{PM}_{2.5}$ samples indicated that soil is the major component of both fractions, while soil contributes more than 50 percent of $\mathrm{PM}_{10}$ 
mass. Sulfate and nitrate account for about 10 percent. Increases in $\mathrm{PM}_{10}$ and $\mathrm{PM}_{2.5}$ mass concentrations are associated with higher concentrations of soil material.

\section{ACKNOWLEDGEMENTS}

Authors thank the U.S. Bureau of Land Management for hosting the mobile trailer inside the fence at Well VH-1 at Crater Flat.

\section{REFERENCES}

Engelbrecht, J.P., I.G. Kavouras, D. Campbell, S.A. Campbell, S. Kohl, and D. Shafer, 2007a. Yucca Mountain Environmental Monitoring Systems Initiative. Air Quality Scoping Study for Ash Meadows National Wildlife Refuge, Nevada. Letter Report DOE/NV/26383-LTR2007-01

Engelbrecht, J.P., I.G. Kavouras, D. Campbell, S.A. Campbell, S. Kohl, and D. Shafer, 2007b. Yucca Mountain Environmental Monitoring Systems Initiative. Air Quality Scoping Study for Beatty, Nevada. Letter Report DOE/NV/26383-LTR2007-02

Engelbrecht, J.P., I.G. Kavouras, D. Campbell, S.A. Campbell, S. Kohl, and D. Shafer, 2007c. Yucca Mountain Environmental Monitoring Systems Initiative. Air Quality Scoping Study for Rachel, Nevada. Letter Report DOE/NV/26383-LTR2007-03

Engelbrecht, J.P., I.G. Kavouras, D. Campbell, S.A. Campbell, S. Kohl, and D. Shafer, 2007d. Yucca Mountain Environmental Monitoring Systems Initiative. Air Quality Scoping Study for Sarcobatus Flats, Nevada. Letter Report DOE/NV/26383-LTR2007-04

Kavouras, I.G., V. Etyemezian, D. DuBois, J. Xu, M. Pitchford, and M. Green, 2005. Assessment of the Principal Causes of Dust-Resultant Haze at IMPROVE Sites in the Western United States. Final report to Western Regional Air Partnership (www.coha.dri.edu/dust).

Lefer, B.L. and R.W. Talbot, 2001. Summertime measurements of aerosol nitrate and ammonium at a northeastern U.S. site. Journal of Geophysical Research, 106, 20,36520,378 .

Malm, W.C., B.A. Schichtel, R.B. Ames, and K.A. Gebhart, 2002. A 10-year spatial and temporal trend of sulfate across the United States. Journal of Geophysical Research, 107, 4627, doi:10.1029/2002JD002107.

Malm, W.C., B.A. Schichtel, M.L. Pitchford, L.L. Ashbaugh, and R.A. Eldred, 2004. Spatial and monthly trends in speciated fine particle concentration in United States. Journal of Geophysical Research, 109, D03306, doi:10.1029/2006JD003739.

White, W.H. and P.T. Roderts, 1977. On the nature and origins of visibility-reducing aerosol in the Los Angeles air basin. Atmospheric Environment, 11, 803-812. 